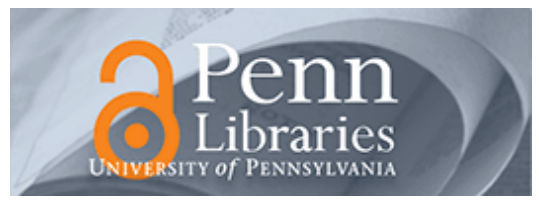

University of Pennsylvania

ScholarlyCommons

Finance Papers

Wharton Faculty Research

2011

\title{
Ambiguous Information, Portfolio Inertia, and Excess Volatility
}

Philipp Karl Illeditsch

University of Pennsylvania

Follow this and additional works at: https://repository.upenn.edu/fnce_papers

Part of the Finance Commons, and the Finance and Financial Management Commons

\section{Recommended Citation}

Illeditsch, P. K. (2011). Ambiguous Information, Portfolio Inertia, and Excess Volatility. The Journal of Finance, 66 (6), 2213-2247. http://dx.doi.org/10.1111/j.1540-6261.2011.01693.x

This paper is posted at ScholarlyCommons. https://repository.upenn.edu/fnce_papers/295

For more information, please contact repository@pobox.upenn.edu. 


\title{
Ambiguous Information, Portfolio Inertia, and Excess Volatility
}

\author{
Abstract \\ I study the effects of risk and ambiguity (Knightian uncertainty) on optimal portfolios and equilibrium \\ asset prices when investors receive information that is difficult to link to fundamentals. I show that the \\ desire of investors to hedge ambiguity leads to portfolio inertia and excess volatility. Specifically, when \\ news is surprising, investors may not react to price changes even if there are no transaction costs or \\ other market frictions. Moreover, I show that small shocks to cash flow news, asset betas, or market risk \\ premia may lead to drastic changes in the stock price and hence to excess volatility. \\ Disciplines \\ Finance | Finance and Financial Management
}




\title{
Ambiguous Information, Portfolio Inertia, and Excess Volatility*
}

\author{
Philipp Karl Illeditsch ${ }^{\dagger}$
}

\author{
March 2011 \\ Journal of Finance, Forthcoming
}

\begin{abstract}
I study the effects of risk and ambiguity (Knightian uncertainty) on optimal portfolios and equilibrium asset prices when investors receive information that is difficult to link to fundamentals. I show that the desire of investors to hedge ambiguity leads to portfolio inertia and excess volatility. Specifically, when news is surprising, then investors may not react to price changes although there are no transaction costs or other market frictions. Moreover, I show that small shocks to cash flow news, asset betas, or market risk premia may lead to drastic changes in the stock price and hence to excess volatility.
\end{abstract}

${ }^{*}$ The previous title of this paper was Ambiguous Information, Risk Aversion, and Asset Pricing.

${ }^{\dagger}$ The Wharton School, University of Pennsylvania, 3620 Locust Walk, 2426 SH-DH, Philadelphia, PA 19104 - 6367, phone: 215 - 898 - 3477, e-mail: pille@wharton.upenn.edu. I am still looking for words to express my gratitude to my dissertation chair Kerry Back. I would like to thank Campbell Harvey (the editor) and an anonymous associated editor and referee for their comments, which substantially improved the paper. I would also like to thank Philip Bond, Domenico Cuoco, Larry Epstein, Michael Gallmeyer, Neal Galpin, Jayant Ganguli, Itay Goldstein, Christian HeyerdahlLarsen, Shane Johnson, Dmitry Livdan, Marcel Rindisbacher, Klaus Ritzberger, Bryan Routledge, Francesco Sangiorgi, Martin Schneider, Nicholas Souleles, Robert Stambaugh, Tommy Stamland, Semih Tartaroglu, Raman Uppal, Julie Wu, Amir Yaron, Motohiro Yogo, Josef Zechner, Stanley Zin, seminar participants at the Mays Business School, the Rotman School of Management, the Stern School of Business, the Wharton School, Carnegie Mellon, the University of Amsterdam, Tilburg University, Warwick Business School, Norwegian School of Economics and Business Administration $(\mathrm{NHH})$, Norwegian School of Management (BI), the Vienna Graduate School of Finance, Columbia University, and participants at the New Stars in Finance Conference in Madrid, the SEDs in Istanbul, the EFA in Bergen, and the Workshop on Risk, Ambiguity, and Decisions in Honor of Daniel Ellsberg in Vienna for helpful comments and suggestions. I am also very grateful for the generous support of the Mays Business School during my Ph.D. education. 
This paper studies the effects of aversion to risk and ambiguity on optimal portfolios and equilibrium asset prices when investors receive public information about the value of an asset. Investors lack data or experience to process this new information and thus do not know the asset distribution when they condition on this information. I show that the desire of investors to hedge against ambiguity (i) leads to portfolio inertia for risky portfolios and (ii) generates excess volatility for asset prices.

Investors face risk and ambiguity when they evaluate an investment in an asset because they neither know the future realization of the asset's payoff (risk), nor the probability of it occurring (ambiguity). This distinction between risk and ambiguity is often attributed to Knight (1921). In this paper, investor's preferences are represented by "max-min" expected utility. In other words, investors evaluate the outcome of an investment with respect to a set of models and then choose the model that leads to the lowest expected utility. These preferences exhibit aversion to ambiguity and are axiomatized by Gilboa and Schmeidler (1989). Moreover, the axioms describe behavior that is consistent with experimental evidence (Ellsberg (1961)) and more recent portfolio choice experiments (Ahn, Choi, Gale, and Kariv (2010) and Bossaerts, Ghirardato, Guarnaschelli, and Zame (2010)).

By focusing on the interaction between risk and ambiguity when investors process a public signal with unknown precision, the contribution of this paper is twofold. First this paper provides novel predictions for optimal portfolios that may help explain why many investors who own stocks do not show much trading activity. For instance, Ameriks and Zeldes (2004) document that almost half of the sample members made no active changes to their portfolio allocation using data from the Surveys of Consumer Finances and from TIAA-CREF.

I show that when news is disappointing, then investors can find risky stock allocations that hedge against ambiguous news and thus it is optimal to stick to 
these allocations even if prices change. Hence, investors exhibit portfolio inertia for risky portfolios without any market frictions such as transaction costs and liquidity needs (Allen and Gale (1994) and Williamson (1994)), entry cost (Vissing-Jorgensen (2002), Paiella (2007), and Yaron and Zhang (2000)), or inattention (Abel, Eberly, and Panageas (2007)). Moreover, portfolio inertia occurs more often when investors use the stock to also hedge the risk of other assets and it is more severe when news is very surprising. ${ }^{1}$

Second, this paper produces new insights into the excess volatility puzzle of stock returns (Shiller (1992)). Specifically, I find that small shocks to cash flow news may result in drastic changes in the interpretation of this news and hence an econometrician observing the resulting large stock price movements may conclude excess volatility. ${ }^{2}$ The amplification of these news shocks is more severe for risky stocks. Similarly, I show that when past evidence about the cash flows of an asset are very disappointing, then small shocks to the beta of the asset with the market portfolio and/or the market risk premium may result in drastic changes in the stock price. Hence, the interaction of risk and ambiguity may help explain that stock price volatility spikes around earnings announcements (Dubinsky and Johannes (2005)) or shortly after very bad news such as the Cuban Missile Crisis, the assassination of JFK, the 9/11 terrorist attacks, or major defaults such as LTCM, Enron, WorldCom, and Lehman Brothers (Bloom (2009)).

This paper is most closely related to Epstein and Schneider (2008), who investigate the impact of ambiguous information on stock prices assuming a representative investor who is risk neutral and averse to ambiguous information. I build on their work along two dimensions: (i) investors are risk averse ${ }^{3}$ and (ii) investors receive stochastic labor income. I show that the interaction of risk and ambiguity leads to portfolio inertia for risky portfolios when investors process ambiguous news. Moreover, I show that risk aversion has qualitatively very different implications for stock 
prices. Specifically, the stock price has a discontinuity which it does not have when investors are risk neutral and thus the effects of ambiguity aversion on stock prices can be distinguished from the effects of risk alone. ${ }^{4}$

It is well known that ambiguity aversion leads to portfolio inertia for the riskfree portfolio and thus helps explain non-market participation (Dow and Werlang (1992), Cao, Wang, and Zhang (2005), and Epstein and Schneider (2007)). Epstein and Schneider (2010) show that portfolio inertia can also arise for risky portfolios when investors use an ambiguous asset to hedge a risky bond position. Epstein and Wang (1994) emphasize that portfolio inertia can arise for long stock positions and thus there may exist a continuum of equilibrium stock prices. I show in this paper that the interaction of risk and ambiguity leads to portfolio inertia for risky portfolios when investors process ambiguous news which leads to an interval of equilibrium stock prices for some fundamentals.

This paper is also related to recent papers by Easley and O'Hara: Easley and O'Hara (2010) show that ambiguity aversion can explain sudden market freezes. In this paper investors are averse to ambiguity in the sense of Bewley (2002) and thus portfolio inertia arises as part of the preference specification. Easley and O'Hara (2009) study the implications of regulation and ambiguity on equilibrium asset prices when some investors are ambiguous about the mean and variance of an asset in the sense of Gilboa and Schmeidler (1989) and some are standard expected utility maximizers. They find that portfolio inertia arises only at the risk-free portfolio and thus ambiguity aversion only indirectly affects equilibrium asset prices. I show in this paper that ambiguity averse investors participate in the stock market but nevertheless exhibit portfolio inertia and thus their behavior directly affects equilibrium asset prices.

Klibanoff, Marinacci, and Mukerji (2005) introduce "smooth" preferences to de- 
scribe ambiguity averse behavior. For instance, Caskey (2009) studies how the aggregation of information affects equity prices when some investors are standard expected utility maximizers and some have smooth ambiguity aversion preferences. I focus on kinked preferences instead of smooth preferences because Ahn, Choi, Gale, and Kariv (2010) and Bossaerts, Ghirardato, Guarnaschelli, and Zame (2010) find that individuals behavior is more easily described with kinked than with smooth preferences. They also document that there is substantial heterogeneity in risk and ambiguity aversion and a non-negligible fraction of individuals behavior is consistent with standard expected utility. Hence, I also study the effects of heterogeneity among investors on equilibrium stock prices and show that the excess volatility result is robust to aggregation.

This paper is also related to the literature on learning about fundamentals to explain the excess volatility puzzle. Brennan and Xia (2001) and Veronesi (2000) consider an economy in which investors learn about the unobservable expected dividend growth rate. In these papers investors have standard expected utility preferences and thus consider a single posterior distribution to determine expected utility. I also study the effects of learning about signal quality on optimal portfolios and equilibrium asset prices when investors are standard expected utility maximizers. I show that asset demand is a strictly decreasing and smooth function of the stock price and thus there is no portfolio inertia. Moreover, the equilibrium stock price is a continuous and smooth function of the signal and thus there is no excess volatility.

Routledge and Zin (2009) and Caballero and Krishnamurthy (2008) study the connection of ambiguity with liquidity. Routledge and Zin (2009) consider a financial intermediary who makes a market in a derivative security and show that ambiguity can drastically increase the bid-ask spread and hence reduce liquidity. Caballero and Krishnamurthy (2008) study the effects of ambiguity about the impact of aggregate liquidity shocks on investors and show that this ambiguity can lead to a socially 
inefficient flight to quality. In this paper, the interaction of risk and ambiguity leads to portfolio inertia and excess volatility.

This paper is organized as follows. In Section I, I introduce the model. In Section II, I solve for optimal asset demand and discuss the portfolio inertia results. In Section III, I solve for equilibrium stock prices. In Section IV, I discuss the robustness of the results to aggregation. In Section V, I study the effects of Bayesian learning on optimal portfolios and equilibrium asset prices and I conclude in Section VI.

\section{Model}

Suppose there are two dates 0 and 1 . Investors can invest in a risk-free asset and a risky asset. Let $p$ denote the price of the risky asset, $\tilde{d}$ the future value or dividend of the risky asset, $\theta$ the number of shares invested in the risky asset, and $\tilde{L}$ the random endowment that investors receive at date 1 . There is no consumption at date zero. The risk-free asset is used as numeraire, so the risk-free rate is zero. Hence, future wealth $\tilde{w}$ is given by

$$
\tilde{w}=w_{0}+(\tilde{d}-p) \theta+\tilde{L}
$$

in which $w_{0}$ denotes initial wealth.

Investors receive a signal $\tilde{s}$ about the future value of the asset $\tilde{d}$. The signal $\tilde{s}$ is conditionally independent of labor income $\tilde{L}$. A model consists of a marginal distribution of $\tilde{d}$ and $\tilde{L}$ and a conditional distribution of $\tilde{s}$ given $\tilde{d}$. Investors neither know the marginal distribution nor the conditional distribution.

I focus in this paper on ambiguity averse investors in the sense of Gilboa and Schmeidler (1989). Specifically, let $u(\cdot)$ denote the Bernoulli utility function of the investor, $m$ a model, $\mathcal{M}(s)$ the set of all models considered by the investor, and $\mathrm{E}_{m}[\cdot]$ the expectation with respect to the belief generated by the model $m$. An ambiguity 
averse investor in the sense of Gilboa and Schmeidler (1989) chooses a portfolio $\theta$ to maximize

$$
\inf _{m \in \mathcal{M}(s)} \mathrm{E}_{m}[u(\tilde{w}) \mid \tilde{s}=s]
$$

subject to the budget constraint given in equation (1).

The curvature of the utility function $u(\cdot)$ determines an investor's risk aversion whereas the size of $\mathcal{M}(s)$ determines an investors aversion to ambiguity. Investors who are averse to ambiguity behave as if they have a set of models (a set of marginals and a set of conditionals) in mind when evaluating the outcome of a decision. The worst case scenario (the belief generated by $m$ that leads to the lowest expected utility) that they consider may depend on the portfolio position $\theta$ and/or the realization of the signal $s$.

Suppose that both the marginal distribution of $\tilde{d}$ and $\tilde{L}$ and the conditional distribution of $\tilde{s}$ given $\tilde{d}$ is normal. There is no ambiguity about the marginal distribution of $\tilde{d}$ and $\tilde{L}$ and thus there is a single normal marginal: ${ }^{5}$

$$
\left(\begin{array}{c}
\tilde{d} \\
\tilde{L}
\end{array}\right) \sim \mathrm{N}\left(\left(\begin{array}{c}
\bar{d} \\
\bar{L}
\end{array}\right),\left(\begin{array}{cc}
\sigma_{d}^{2} & \rho \sigma_{d} \sigma_{L} \\
\rho \sigma_{d} \sigma_{L} & \sigma_{L}^{2}
\end{array}\right)\right)
$$

Investors are ambiguous about the conditional distribution of $\tilde{s}$ given $\tilde{d}$ and thus there is a family of conditionals:

$$
\tilde{s}=\tilde{d}+\tilde{\varepsilon}, \quad \tilde{\varepsilon} \sim \mathrm{N}\left(0, \sigma^{2}\right)
$$

in which $\sigma^{2} \in\left[\sigma_{a}^{2}, \sigma_{b}^{2}\right] \subset[0, \infty]$. Investors are ambiguous about the precision of the signal and thus consider a set of precisions when processing this signal. The signal is conditionally independent of labor income and thus $\operatorname{Cov}[\tilde{\varepsilon}, \tilde{L}]=0$.

I follow Gilboa and Schmeidler (1993) and assume that investors update their 
beliefs model by model using Bayes rule. Hence, observing the signal does not lead to a reduction in ambiguity and $\mathcal{M}(s)=\mathcal{M}{ }^{6}$ Specifically, each model $m \in \mathcal{M}$ determines a conditional belief for $\tilde{d}$ and $\tilde{L}$ given $\tilde{s}$ and hence standard normal-normal updating for each $\sigma^{2} \in\left[\sigma_{a}^{2}, \sigma_{b}^{2}\right]$ leads to

$$
\left(\begin{array}{c}
\tilde{d} \\
\tilde{L}
\end{array}\right) \mid \tilde{s}=s \sim \mathrm{N}_{\phi}\left(\left(\begin{array}{c}
\bar{d}+\phi(s-\bar{d}) \\
\bar{L}+z \phi(s-\bar{d})
\end{array}\right),\left(\begin{array}{cc}
\sigma_{d}^{2}(1-\phi) & \rho \sigma_{d} \sigma_{L}(1-\phi) \\
\rho \sigma_{d} \sigma_{L}(1-\phi) & \sigma_{L}^{2}\left(1-\phi \rho^{2}\right)
\end{array}\right)\right)
$$

in which $z=\rho \frac{\sigma_{L}}{\sigma_{d}}$ and $\phi=\sigma_{d}^{2} /\left(\sigma_{d}^{2}+\sigma^{2}\right)$. If labor income is correlated with the asset $(z \neq 0)$, then investors use the signal to learn about future labor income which generates ambiguity about the distribution of future labor income.

It is convenient to describe the informativeness of the signal by $\phi$ and hence the set of posterior beliefs is given by $\left[\phi_{a}, \phi_{b}\right] \subset[0,1]$ with

$$
\begin{aligned}
& \phi_{a}=\sigma_{d}^{2} /\left(\sigma_{d}^{2}+\sigma_{b}^{2}\right) \\
& \phi_{b}=\sigma_{d}^{2} /\left(\sigma_{d}^{2}+\sigma_{a}^{2}\right) .
\end{aligned}
$$

The utility of an investor who is averse to ambiguous information holds $\theta$ shares of the risky asset and receives the random endowment $\tilde{L}$ is therefore ${ }^{7}$

$$
\min _{\phi \in\left[\phi_{a}, \phi_{b}\right]} \mathrm{E}_{\phi}\left[u\left(w_{0}+(\tilde{d}-p) \theta+\tilde{L}\right) \mid \tilde{s}=s\right]
$$

Investors are more averse to ambiguous information if the interval $\left[\phi_{a}, \phi_{b}\right]$ is large and therefore the degree of aversion to ambiguous information can be measured by $\phi_{b}-\phi_{a}$. 


\section{Optimal Demand}

In this section I determine the optimal portfolio of an investor who can invest in a risky asset and a risk-free asset and who receives stochastic labor income and an ambiguous signal about the future value of the risky asset. I show that there is a range of prices for which investors do not change their stock position and hence exhibit portfolio inertia.

Suppose investors have CARA utility over future wealth $\tilde{w}$ (i.e. $u(\tilde{w})=-e^{-\gamma \tilde{w}}$ with $\gamma>0$ ) and let $\operatorname{CE}(\theta)$ denote the certainty equivalent of an ambiguity averse investor. Then the investor's utility given in equation (8) is equal to $u(\mathrm{CE}(\theta))$ with $^{8}$

$$
\mathrm{CE}(\theta)=\min _{\phi \in\left[\phi_{a}, \phi_{b}\right]}\left(\mathrm{E}_{\phi}[\tilde{w} \mid \tilde{s}=s]-\frac{1}{2} \gamma \operatorname{Var}_{\phi}[\tilde{w} \mid \tilde{s}=s]\right)
$$

The assumption of CARA-utility and normal beliefs leads to mean-variance preferences over future wealth (financial wealth plus labor income) in which the informativeness of the signal measured by $\phi$ affects both the mean and the variance. The worst case scenarios for the mean and the variance can not be chosen independently of each other and hence the belief of an ambiguity averse investor will depend on the realization of the signal s, the portfolio position $\theta$, and the beta of labor income with the asset $z$ as the next proposition shows. ${ }^{9}$

Proposition 1 (Preferences). Let $\hat{\theta} \equiv 2(\bar{d}-s) /\left(\gamma \sigma_{d}^{2}\right)$. Then,

$$
C E(\theta)= \begin{cases}\mathrm{E}_{\phi_{a}}[\tilde{w} \mid \tilde{s}=s]-\frac{1}{2} \gamma \operatorname{Var}_{\phi_{a}}[\tilde{w} \mid \tilde{s}=s] & \text { if } \quad \theta+z \leq \min (\hat{\theta}, 0) \\ \mathrm{E}_{\phi_{b}}[\tilde{w} \mid \tilde{s}=s]-\frac{1}{2} \gamma \operatorname{Var}_{\phi_{b}}[\tilde{w} \mid \tilde{s}=s] & \text { if } \min (\hat{\theta}, 0)<\theta+z<\max (\hat{\theta}, 0) \\ \mathrm{E}_{\phi_{a}}[\tilde{w} \mid \tilde{s}=s]-\frac{1}{2} \gamma \operatorname{Var}_{\phi_{a}}[\tilde{w} \mid \tilde{s}=s] & \text { if } \quad \theta+z \geq \max (\hat{\theta}, 0) .\end{cases}
$$

The certainty equivalent $C E(\theta)$ is a continuous and concave function of the stock demand $\theta$. Moreover, it is continuously differentiable except for the portfolios $\theta=-z$ 
and $\theta=\hat{\theta}-z$ if $s \neq \bar{d}$.

To gain some more intuition consider an investor who is contemplating a long position in the asset $(\theta>0)$. The worst case scenario for the posterior asset mean is a reliable signal (high $\phi$ ) if bad news arrives and an unreliable signal (low $\phi$ ) if good news arrives because the mean is significantly adjusted downwards with bad news and moderately adjusted upwards with good news.

Similarly, the worst case scenario for the posterior asset mean when an investor is contemplating a short position in the asset $(\theta<0)$ is an unreliable signal when bad news arrives and a reliable signal when good news arrives. On the other hand, the worst case scenario for the residual asset variance is always an unreliable signal because in this case less risk is resolved by the signal.

Suppose there is no correlation between the asset and labor income $(z=0)$ and hence there is no ambiguity about future labor income. If the signal conveys bad news, then a reliable signal decreases the posterior asset mean (since more weight is put on the signal) but it also reduces the residual variance (since more risk is resolved by the signal). Investors are more worried about the posterior mean for small risks and are more worried about the residual variance for big risks. Hence, the signal is treated as reliable $\left(\phi=\phi_{b}\right)$ for moderate long positions in the asset $(0<\theta<\hat{\theta})$ and as unreliable $\left(\phi=\phi_{a}\right)$ otherwise.

There is a portfolio with an intermediate level of risk $(\theta=\hat{\theta})$ for which the counteracting mean and variance effects offset each other and utility becomes independent of signal precision. This portfolio is more risky if there is a big news surprise because in this case there is more ambiguity about the mean. It is less risky when investors are more risk averse because in this case investors are more worried about the ambiguous variance. The second portfolio for which utility does not depend on signal precision is the riskless portfolio $\theta=0$. 
Suppose labor income is correlated with the asset $(z \neq 0)$ and thus there is also ambiguity about future labor income. Then the portfolios for which utility is independent of the precision of the signal are shifted by the hedging demand for labor income $z$ and therefore are $\theta=-z$ and $\theta=\hat{\theta}-z$.

To summarize, an investor who has CARA utility and is averse to ambiguous information in the sense of Gilboa and Schmeidler (1989) will evaluate the outcome of a portfolio with respect to the belief $\phi$ that leads to the lowest expected utility. Hence, utility of an ambiguity averse investor has two kinks if the signal doesn't confirm the unconditional mean of the dividend and are otherwise smooth. ${ }^{10}$ The optimal demand for the risky asset when investors are averse to risk and ambiguous information is determined below.

\section{A Savage Benchmark}

The demand of a standard expected utility maximizer in the sense of Savage (1954) with subjective belief $\phi$ is given in the next proposition.

Proposition 2 (Optimal Demand-Savage Benchmark). Let $\phi_{a}=\phi_{b}=\phi$. Then the demand function for the risky asset is

$$
\theta_{\phi}(p)=\frac{\mathrm{E}_{\phi}[\tilde{d} \mid \tilde{s}=s]-p}{\gamma \operatorname{Var}_{\phi}[\tilde{d} \mid \tilde{s}=s]}-z .
$$

Investors use the signal to learn about the future value of the asset. Suppose there is no correlation between the endowment and the asset; i.e. $z=0$. Then investors will buy the asset as long as the posterior mean exceeds the asset price and they will sell otherwise. Moreover, investors will buy/sell larger quantities if the signal is more precise. If investors receive an endowment that is correlated with the asset $(z \neq 0)$, 
then they will use the asset to hedge their labor income risk. For instance, investors will sell some of the asset if their future labor income is positively correlated with the asset $(z>0)$ and buy otherwise $(z<0)$.

\section{B Ambiguity Averse Investors}

Let $\theta_{a}(p)$ denote the demand of a Savage investor with subjective belief $\phi_{a}$ (a standard expected utility maximizer who treats the signal as unreliable) and $\theta_{b}(p)$ the demand of a Savage investor with subjective belief $\phi_{b}$ (a standard expected utility maximizer who treats the signal as reliable). The solution to the portfolio choice problem of an investor who has CARA utility and is averse to ambiguity is provided in the next theorem.

Theorem 1 (Optimal Demand). The optimal demand function $\theta(p)$ for an investor with risk aversion $\gamma$ and aversion to ambiguous information described by $\left[\phi_{a}, \phi_{b}\right]$ is continuously decreasing in $p$ if $s=\bar{d}$ and continuously non-increasing in $p$ if $s \neq \bar{d}$. Specifically, let $\hat{\theta}=2(\bar{d}-s) /\left(\gamma \sigma_{d}^{2}\right)$ then

$$
\theta(p)=\left\{\begin{array}{lr}
\theta_{a}(p) & \leq p_{1} \equiv \mu_{\phi_{a}}(s)-\max (\hat{\theta}, 0) \gamma v_{\phi_{a}} \\
\max (\hat{\theta}, 0)-z & p_{1}<p \leq p_{2} \equiv \mu_{\phi_{b}}(s)-\max (\hat{\theta}, 0) \gamma v_{\phi_{b}} \\
\theta_{b}(p) & p_{2}<p \leq p_{3} \equiv \mu_{\phi_{b}}(s)-\min (\hat{\theta}, 0) \gamma v_{\phi_{b}} \\
\min (\hat{\theta}, 0)-z & p_{3}<p \leq p_{4} \equiv \mu_{\phi_{a}}(s)-\min (\hat{\theta}, 0) \gamma v_{\phi_{a}} \\
\theta_{a}(p) & p>p_{4},
\end{array}\right.
$$

in which $\mu_{\phi}(s)=\bar{d}+\phi(s-\bar{d})$ denotes the posterior asset mean and $v_{\phi}=\sigma_{d}^{2}(1-\phi)$ denotes the residual asset variance when the signal precision is $\phi$.

Suppose the asset is uncorrelated with labor income $(z=0)$. Optimal demand when the signal conveys bad news $(s<\bar{d})$ is presented in the left graph of Figure 1. If 
ambiguity averse investors (solid line) take on large long positions in the asset, then they are more worried about risk than a low posterior mean and hence they treat the signal as uninformative. In this case their demand coincides with the demand of a Savage investor with belief $\phi_{a}$ (dashed line). However, if they take on a moderate long position when the signal conveys bad news, then they are more worried about a low posterior mean than risk and hence they treat the signal as informative. In this case their demand coincides with the demand of a Savage investor with belief $\phi_{b}$ (chain-dotted line). ${ }^{11}$ There are two portfolios $\theta=0$ and $\theta=\hat{\theta}$ for which the demand of an ambiguity averse investor is insensitive to changes in the asset price and thus it is distinctly different from the demand of a Savage investor.

\section{*** INSERT FIGURE 1 ABOUT HERE ***}

\section{Portfolio Inertia}

It is well known in the literature that with max-min preferences portfolio inertia arises at certainty (Dow and Werlang (1992), Cao, Wang, and Zhang (2005), and Epstein and Schneider (2007)). For instance, if investors think about taking on a small long (short) position in the asset, then they fear reliable signals that convey bad (good) news and unreliable signals that convey good (bad) news because this means a low posterior mean for their stock position and hence there is a range of prices for which they rather not be invested in the stock to avoid ambiguity.

Ambiguous information leads to inertia away from certainty because a reliable signal that conveys bad news decreases the posterior mean but also reduces the residual variance. The counteracting effect of mean and variance on utility exactly offset each other and utility becomes independent of signal precision if investors long the

portfolio $\theta=\hat{\theta}$. This portfolio hedges against ambiguity and small price changes cannot entice investors away from it because taking on slightly more or less risk than 
that of the hedging portfolios leads to a large change in the worst case scenario belief about the informativeness of the signal. ${ }^{12}$

The right graph of Figure 1 shows optimal demand when the signal conveys bad news $(s<\bar{d})$ and asset and labor income are positively correlated $(z>0)$. In this case the hedging demand for labor income risk is negative and demand shifts downwards. Neither the posterior mean nor the residual variance of future wealth (financial wealth and labor income) depend on the signal precision for the portfolios $\theta=-z$ and $\theta=\hat{\theta}-z$ and thus investors exhibit portfolio inertia for two risky portfolios. The portfolios that hedge against ambiguity are less invested in the stock because investors need to sell some of the stock to reduce the additional ambiguity about future wealth due to the positive correlation between the asset and labor income.

The size of the inaction region depends on the degree of ambiguity and on the news surprise as the next proposition shows.

Proposition 3 (Portfolio Inertia). The size of the price region at which investors exhibit portfolio inertia is

$$
\left(\phi_{b}-\phi_{a}\right)|s-\bar{d}|
$$

There is more ambiguity about the posterior mean when news is very surprising and thus larger price changes are necessary to convince an ambiguity averse investor to give up either of the two hedging portfolios $\theta=-z$ and $\theta=\hat{\theta}-z$. On the other hand, if the signal confirms the unconditional mean of the dividend, then there is no ambiguity about the conditional mean and hence there is no portfolio inertia because the ambiguity averse investor treats the signal as unreliable for all portfolios and hedging demands for labor income.

The left graph of Figure 1 shows optimal demand when the signal conveys bad news and the right graph shows optimal demand when the signal conveys good news. 
A very large news surprise leads to a large inaction region (dashed line). Moreover, the portfolio position at which investors exhibit inertia is riskier when news is very surprising because the resulting increase in ambiguity about the residual variance is offset by the increase in ambiguity about the posterior mean when news is worse than expected.

\section{*** INSERT FIGURE 2 ABOUT HERE ***}

I conclude this section with a summary of the predictions for optimal portfolios.

Corollary 1. If investors are averse to risk and ambiguity, then

(i) there is a range of prices for which investors are neither long/nor short the stock when news is surprising,

(ii) there is a range of prices for which investors do not adjust their long stock position when news is worse than expected,

(iii) there is a range of prices for which investors do not adjust their short stock position when news is better than expected,

(iv) the need of investors to hedge labor income risk leads to portfolio inertia for risky portfolios when news is surprising, and

(v) portfolio inertia is more severe for extreme news surprises.

\section{Equilibrium}

In this section I solve for equilibrium prices when a representative investor receives ambiguous information about an asset. I show that small shocks to news about cash flows, asset betas, or market risk premia may lead to drastic changes in asset prices. The proof of the existence of a representative investor is deferred to Section IV. 
Suppose there is a representative investor with CARA-utility who is averse to ambiguity. In equilibrium, the representative investor holds the stock and consumes

the liquidating dividend $\tilde{d}$ and the labor income $\tilde{L}$. Hence, the stock price at date one equals the liquidating dividend and $\theta=1$. The stock price at date zero which is denoted by $p(s)$ is determined below.

\section{A Savage Benchmark}

Suppose the representative investor is a standard expected utility maximizer with subjective belief $\phi$ and let $p_{\phi}(s)$ denote the stock price in this case. The equilibrium stock price is provided in the next proposition.

Proposition 4 (Equilibrium Price-Savage Benchmark). Let $\phi_{a}=\phi_{b}=\phi$. Then

$$
p_{\phi}(s)=\mathrm{E}_{\phi}[\tilde{d} \mid \tilde{s}=s]-\gamma(1+z) \operatorname{Var}_{\phi}[\tilde{d} \mid \tilde{s}=s]
$$

The stock price is lower when labor income is positively correlated with the aggregate dividend because in this case the representative investor would like to sell the stock to hedge labor income risk and thus the price has to decrease to keep him from doing that. Similarly, better news increases the posterior mean of the stock and thus the price has to increase to clear the market.

The stock price satisfies the Capital Asset Pricing Model:

$$
\begin{aligned}
p_{\phi}(s) & =\mathrm{E}_{\phi}[\tilde{d} \mid \tilde{s}=s]-\gamma \operatorname{Cov}_{\phi}[\tilde{w}, \tilde{d} \mid \tilde{s}=s] \\
& =\bar{d}+\phi(s-\bar{d})-(1-\phi) \beta \lambda,
\end{aligned}
$$

in which $\beta$ denotes the unconditional beta of the stock with the market portfolio and 
$\lambda$ denotes the unconditional risk premium of the market portfolio. ${ }^{13}$ Specifically,

$$
\begin{aligned}
\beta & =\frac{\operatorname{Cov}[\tilde{w}, \tilde{d}]}{\operatorname{Var}[\tilde{w}]}=\frac{(1+z) \sigma_{d}^{2}}{\sigma_{d}^{2}+2 \rho \sigma_{d} \sigma_{L}+\sigma_{L}^{2}} \\
\lambda & =\gamma \operatorname{Var}[\tilde{w}]=\gamma\left(\sigma_{d}^{2}+2 \rho \sigma_{d} \sigma_{L}+\sigma_{L}^{2}\right) .
\end{aligned}
$$

The stock price $p_{\phi}(s)$ is a strictly increasing continuous function of the signal $s$ and a strictly decreasing and continuous function of the unconditional stock risk premium $\beta \lambda$. This is no longer true when investors are averse to ambiguity as the remainder of this section shows.

\section{B Aversion to Ambiguity}

Let $p_{a}(s)$ and $p_{b}(s)$ denote the equilibrium stock price when the representative investor is a standard expected utility maximizers with subjective belief $\phi_{a}$ and $\phi_{b}$, respectively. The equilibrium stock price when the representative investor is averse to ambiguity is given in the next theorem. ${ }^{14}$

Theorem 2 (Equilibrium Stock Price). Let $\hat{s}=\bar{d}-\frac{1}{2} \beta \lambda$. There is a unique equilibrium stock price correspondence:

$$
p(s) \in\left\{\begin{array}{llllll}
\left\{p_{a}(s)\right\} & \text { if } s>\hat{s} \text { and } \beta>0 & \text { or } s<\hat{s} & \text { and } & \beta<0 \\
\mathcal{P}_{\hat{s}} & \text { if } s=\hat{s} & \text { and } \beta>0 & \text { or } & \beta<0 \\
\left\{p_{b}(s)\right\} & \text { if } s<\hat{s} & \text { and } \beta>0 & \text { or } s>\hat{s} & \text { and } & \beta<0 \\
\mathcal{P}_{0}(s) & \text { if } & & & & \\
& & &
\end{array}\right.
$$

Specifically, $p \in \mathcal{P}_{\hat{s}}$, if $\exists \phi \in\left[\phi_{a}, \phi_{b}\right]$ such that $p=\bar{d}+\phi(\hat{s}-\bar{d})-(1-\phi) \beta \lambda$ and $p \in \mathcal{P}_{0}(s)$, if $\exists \phi \in\left[\phi_{a}, \phi_{b}\right]$ such that $p=\bar{d}+\phi(s-\bar{d})$.

The left graph of Figure 3 shows the equilibrium signal-to-price map when there 
is no ambiguity about future labor income $\left(z=0\right.$ and thus $\left.\beta \lambda=\gamma \sigma_{d}^{2}\right)$. The representative investor is more worried about a low posterior mean when the signal is lower than $\hat{s}$ and more worried about risk when the signal exceeds $\hat{s}$ because the posterior mean goes down when the signal decreases, whereas the residual variance does not depend on the signal. Hence, there is a strong price reaction to very bad news because the representative investor treats all signals below $\hat{s}$ as reliable. ${ }^{15}$ Moreover, the price suddenly drops when the signal increase through the critical value $\hat{s}$, because switching from a reliable to an unreliable signal leads to a sudden increase in the risk premium and thus to a sudden drop in the stock price.

\section{*** INSERT FIGURE 3 ABOUT HERE ***}

What is the equilibrium price at $s=\hat{s}$ ? Let $p^{+}(\hat{s}) \equiv \lim _{s \downarrow \hat{s}} p(s)$ denote the limit of $p(s)$ when $s$ approaches $\hat{s}$ from the right and $p^{-}(\hat{s}) \equiv \lim _{s \uparrow \hat{s}} p(s)$ denote the limit of $p(s)$ when $s$ approaches $\hat{s}$ from the left. Then every price that is contained in the interval $\left[p^{+}(\hat{s}), p^{-}(\hat{s})\right]$ is an equilibrium. Intuitively, if $s=\hat{s}$, then utility of the representative investor does not depend on the precision of the signal at the market clearing stock demand $\theta=1$ and thus there is no ambiguity. The representative investor is averse to ambiguity and thus there is an interval of equilibrium prices for which she does not give up the portfolio $\theta=1$ that hedges against ambiguity.

\section{Shocks to Cash Flow News}

The sudden change in the representative investor's worst case scenario belief amplifies the effects of news shocks on stock prices and hence leads to excess volatility. Specifically, supply (dotted line in the right graph of Figure 3) is price inelastic and equal to demand for an interval of prices at the critical signal value $\hat{s}$ (solid line). A slightly lower signal value shifts the inaction region of the demand upwards (chain dotted 
line) and thus pushes the (now unique) equilibrium price close to $p^{-}(\hat{s})$ whereas a slightly higher signal value shifts the inaction region downwards (dashed line) and pushes the new equilibrium price close to $p^{+}(\hat{s}) .{ }^{16}$ Hence, small changes to the signal can result in large changes in the asset price.

To quantify the amplification mechanism of small news shocks I compute the variance of the asset price conditional on the signal being in an $\epsilon$-intervall around the critical signal value $\hat{s}$. The results are plotted in the left graph of Figure 4 when the "true" conditional distribution is characterized by $\phi=\left(\phi_{a}+\phi_{b}\right) / 2 .{ }^{17}$ The variance is small when investors are neutral to risk or ambiguity but not when they are averse to both. Intuitively, there is no discontinuity in the equilibrium signal-to-price map if investors are standard expected utility maximizers $\left(\phi_{a}=\phi_{b}=\phi\right)$ or if they are risk neutral $(\gamma=0)$. The right graph of Figure 4 shows the corresponding equilibrium signal-to-price map for a Savage investor with belief $\phi=\left(\phi_{a}+\phi_{b}\right) / 2$, a risk neutral ambiguity averse investor, and two investors who are averse to risk and ambiguity. ${ }^{18}$

\section{*** INSERT FIGURE 4 ABOUT HERE ***}

The amplification of news shocks occurs when the signal is close to the critical signal value $\hat{s}=\bar{d}-\frac{1}{2} \beta \lambda$ in which case utility of the representative investor is independent of signal precision and thus she is perfectly hedged against ambiguity. This is formalized in the next proposition by computing the size of the price discontinuity.

Proposition 5 (Shocks to Cash Flow News). Let $\hat{s}=\bar{d}-\frac{1}{2} \beta \lambda$. Then

$$
\Delta p(\hat{s})=\lim _{s \downarrow \hat{s}} p(s)-\lim _{s \uparrow \hat{s}} p(s)=-\frac{1}{2}\left(\phi_{b}-\phi_{a}\right)(\max (\beta, 0)-\min (\beta, 0)) \lambda .
$$

The critical signal value $\hat{s}$ is decreasing in the unconditional risk premium of the asset $(\beta \lambda)$ because in this case investors are more worried about risk and thus the news has to be more surprising to avoid a decrease in the risk of the portfolio that 
hedges against ambiguity. Hence, price crashes for risky stocks only occur for big news surprises. But for big news surprises the difference between the conditional risk premium of reliable and unreliable signals is large and thus prices crash more for risky stocks. This is illustrated in the graphs of 5 that show the equilibrium signal-to-price map for positive, zero, and negative beta stocks.

\section{*** INSERT FIGURE 5 ABOUT HERE ***}

The predictions for the effects of shocks to cash flow news on stock prices are summarized in the next corollary.

Corollary 2. If investors are averse to risk and ambiguity then

(i) small shocks to cash flow news may lead to drastic changes in the price of positive beta stocks if the news is disappointing,

(ii) small shocks to cash flow news may lead to drastic changes in the price of negative beta stocks if the news is good,

(iii) and the amplification of news shocks is stronger for riskier stocks.

\section{Shocks to Asset Betas and Market Risk Premia}

So far we have only looked at how changes in cash flow news affect equilibrium stock prices. I will now take the news about cash flows as given and study how changes to asset betas and/or market risk premia (and thus shocks to the stock risk premium) will effect stock prices.

Suppose investors receive ambiguous news about the future dividend of the stock. The equilibrium price determined in Theorem 2 can be also seen as a mapping from the unconditional stock risk premium $\beta \lambda$ to the equilibrium price $p(\beta \lambda) .{ }^{19}$ The left 
graph of Figure 6 shows the equilibrium price mapping for bad news and the right graph shows it for good news.

There is an interval of equilibrium stock prices if $\beta \lambda=0$ or $\beta \lambda=\widehat{\beta \lambda} \equiv 2(\bar{d}-s)$ because in this case the representative investor's utility does not depend on the precision of the signal and thus there is no ambiguity about the market portfolio. A change in the beta of the stock changes the hedging demand for labor income risk $(z)$ and thus affects the mean and the variance of the market portfolio. Suppose news is worse than expected (left graph). Then the representative investors treats the signal as reliable for moderate stock risk premia $(0<\beta \lambda<\widehat{\beta \lambda})$ and as unreliable otherwise. If the risk premium increases through either of the two critical values than the representative investor dramatically changes the worst case scenario belief and thus an econometrician who observes the resulting drastic price change may conclude excess volatility.

\section{*** INSERT FIGURE 6 ABOUT HERE ***}

The amplification mechanism for shocks to stock risk premia is quantified in the next proposition.

Proposition 6 (Shocks to Stock Risk Premia). Fix $s \in \mathcal{R}$ and define $\widehat{\beta \lambda}=2(\bar{d}-s)$. Then

$$
\begin{aligned}
\Delta p(\widehat{\beta \lambda}) & =\lim _{\beta \lambda \downarrow \downarrow} p(\beta \lambda)-\lim _{\beta \lambda \uparrow \widehat{\beta \lambda}} p(\beta \lambda) \\
& =-\left(\phi_{b}-\phi_{a}\right)(\max (\bar{d}-s, 0)-\min (\bar{d}-s, 0))
\end{aligned}
$$

and

$$
\begin{aligned}
\Delta p(0) & =\lim _{\beta \lambda \downarrow 0} p(\beta \lambda)-\lim _{\beta \lambda \uparrow 0} p(\beta \lambda) \\
& =-\left(\phi_{b}-\phi_{a}\right)(\max (\bar{d}-s, 0)-\min (\bar{d}-s, 0)) .
\end{aligned}
$$


If news is very surprising than there is more ambiguity about the posterior mean and hence there is a bigger difference between the stock price for reliable and unreliable signals. I conclude this section with a summary of the predictions for the effects of shocks to asset betas and/or market risk premia on stock prices.

Corollary 3. If investors are averse to risk and ambiguity and cash flow news is surprising, then

(i) small shocks to asset betas may lead to drastic changes in stock prices,

(ii) small shocks to market risk premia may lead to drastic changes in stock prices, (iii) and amplification of these shocks is more severe when news is more surprising.

\section{Aggregation}

So far I have studied properties of equilibrium stock prices when the economy is populated by a single representative investors. However, Ahn, Choi, Gale, and Kariv (2010) and Bossaerts, Ghirardato, Guarnaschelli, and Zame (2010) document that there is a lot of heterogeneity in aversion to risk and ambiguity. Moreover, Chapman and Polkovnichenko (2009) demonstrate that the size of the market risk premium is sensitive to ignoring heterogeneity because of the endogenous withdrawal of ambiguity averse investors from the market for the risky asset. Similarly, Easley and O'Hara (2009) find that ambiguity averse investors only indirectly affect equilibrium asset

prices because when they invest in the stock market then they behave like standard expected utility maximizer.

This raises the following three questions that will be addressed in this section: ${ }^{20}$

(i) Does there exist a representative investor with the properties assumed in Section 
III? Yes, there does exist a representative investor if all investors have the same ambiguity.

(ii) If there does not exist a representative investor, is there still a discontinuity in the equilibrium signal-to-price map? If investors differ with respect to their aversion to ambiguity, then there is still a discontinuity in the equilibrium signalto-price map if the range of signal precisions considered by investors overlap.

(iii) Do ambiguity averse investors directly effect asset prices in equilibrium? In other words, is there an equilibrium in which ambiguity averse investors invest in the stock and behave distinctly different from standard expected utility maximizers? Yes, ambiguity averse investors directly affect equilibrium asset prices.

Suppose there is no labor income and consider three investors that differ with respect to their aversion to risk and ambiguity. The first investor has the highest degree of aversion to risk and ambiguity $\left(\gamma_{1}=5\right.$ and $\left.\left[\phi_{a 1}, \phi_{b 1}\right]=[1 / 10,9 / 10]\right)$. Her demand (dotted line) does not change for a wide range of prices and is only moderately decreasing otherwise. The second investor (chain-dotted line) has the lowest degree of aversion to ambiguity $\left(\gamma_{2}=5 / 4\right.$ and $\left.\left[\phi_{a 2}, \phi_{b 2}\right]=[1 / 4,3 / 4]\right)$, whereas the third investor (dashed line) has the lowest degree of risk aversion $\left(\gamma_{3}=1\right.$ and $\left.\left[\phi_{a 3}, \phi_{b 3}\right]=[1 / 4,4 / 5]\right)$. The left graph of Figure 7 shows the demand function for all three investors and aggregate demand.

\section{*** INSERT FIGURE 7 ABOUT HERE ***}

There is a range of prices for which aggregate demand (solid line) is flat. The individual demand for the second investor (chain-dotted line) is constant equal to $2 / 5$ for the same range of prices whereas the demand for the third investor (dashed line) is $1 / 2$ and the demand for the first investor (dotted line) is $1 / 10$ for an even wider range 
of prices. Hence, aggregate demand sums up to the market clearing stock demand of one and the range of prices at which the investor with the smallest aversion to ambiguity does not change her demand comprises the interval of equilibrium prices.

The right graph of Figure 7 shows the equilibrium signal-to-price map when the economy consists of the three investors with different aversion to risk and ambiguity described above (solid line). There is a price discontinuity at the critical signal value $\hat{s}=\bar{d}-\gamma \sigma_{d}^{2} / 2=93.75$ in which $1 / \gamma$ is the sum of the risk tolerance of the three investor $\left(1 / \gamma=\sum_{h=1}^{3} 1 / \gamma_{h}=2\right)$. Moreover, there is a range of prices $\left[p_{1}, p_{2}\right]=[89.1,92.2]$ for which all three investors don't change their long stock positions and thus behave distinctly different from standard expected utility maximizers.

Consider the same three investors but assume that they all have the same ambiguity $\left[\phi_{a}, \phi_{b}\right]=[1 / 4,3 / 4]$. In this case there exists a representative investor with risk tolerance equal to the sum of the risk tolerances of all three investors. ${ }^{21}$ The dashed line in the right graph of Figure 7 shows the equilibrium signal-to-price map in this case. If the economy is populated by the three heterogeneous investors, then the price reacts more to signals lower than $\hat{s}$ and less to signals larger than $\hat{s}$ because there is more ambiguity than in the economy with the representative investor.

The size of the price discontinuity when there is heterogeneity with respect to ambiguity is determined by the investor with the lowest degree of ambiguity. This implies that there is no discontinuity in the equilibrium signal-to-price map if at least one investor has standard expected utility preferences. ${ }^{22}$ However, the next example shows that even with no discontinuity, prices may still abruptly change because ambiguity averse investors directly affect prices when they change the worst case scenario belief in equilibrium.

For instance, consider an economy in which one investor is averse to ambiguity and the other investor is not. The ambiguity averse investor has the range of beliefs 
$\left[\phi_{a}, \phi_{b}\right]=[1 / 4,3 / 4]$ and risk aversion $\gamma=1$. The Savage investor has the same risk aversion and the subjective belief $\phi_{m}=\left(\phi_{a}+\phi_{b}\right) / 2=1 / 2$. The equilibrium signal-to-price map is shown by the solid line in the right graph of Figure 8. For comparison, this graph also shows the equilibrium price when the economy consists of two Savage investors with subjective beliefs $\phi_{m}$ and $\phi_{b}$ (dashed line) and $\phi_{m}$ and $\phi_{a}$ (chain-dotted), respectively.

The solid line in the left graph shows the equilibrium demand of the ambiguity averse investor and the dashed line shows the equilibrium demand of the Savage investor with belief $\phi_{m}$. There is a range of signal values for which (i) both investors are in the market and the ambiguity averse investor behaves like a Savage investor, (ii) both investors are in the market and the ambiguity averse investor does not behave like a Savage investor, (iii) the ambiguity averse investor does not participate and does not behave like a Savage investor (this is not apparent from the figure but occurs for very bad news and very good news).

\section{*** INSERT FIGURE 8 ABOUT HERE ***}

\section{Bayesian Model Uncertainty}

I have shown in the previous sections that investors who are averse to risk and ambiguity hedge against uncertain information quality and thus exhibit portfolio inertia. This behavior leads to an amplification mechanism of small shocks to cash flow news and risk premia for equilibrium asset prices. These results are not obtained when investors are standard expected utility maximizers with known information quality.

I consider in this section investors who maximize standard expected utility and have a unique prior over the range of signal precisions $\left[\phi_{a}, \phi_{b}\right]$ and thus also benefit from hedging uncertainty about information quality. I show that optimal demand 
of these investors is a strictly decreasing and smooth function of the stock price and hence there is no portfolio inertia. Moreover, the equilibrium stock price is a smooth function of the signal and hence there is no excess volatility. ${ }^{23}$

Consider the model described in Section I without the random endowment. The precision of the signal is random and denoted by $\tilde{\phi}$. It can can take on either the value $\phi_{a}$ or $\phi_{b}$ with equal probability. Investors choose $\theta$ to maximize

$$
\mathrm{E}\left[u\left(w_{0}+(\tilde{d}-p) \theta\right) \mid \tilde{s}=s\right]=\pi_{s} u\left(\mathrm{CE}^{S}\left(\theta, \phi_{a}\right)\right)+\left(1-\pi_{s}\right) u\left(\mathrm{CE}^{S}\left(\theta, \phi_{b}\right)\right),
$$

in which

$$
\mathrm{CE}^{S}(\theta, \phi)=w_{0}+(\mathrm{E}[\tilde{d} \mid \tilde{s}=s, \tilde{\phi}=\phi]-p) \theta-\frac{1}{2} \gamma \operatorname{Var}[\tilde{d} \mid \tilde{s}=s, \tilde{\phi}=\phi] \theta^{2}
$$

and

$$
\pi_{s} \equiv \operatorname{Prob}\left(\tilde{\phi}=\phi_{a} \mid \tilde{s}=s\right)=\left(1+\sqrt{\frac{\phi_{b}}{\phi_{a}}} e^{-\frac{1}{2}\left(\phi_{b}-\phi_{a}\right)\left(\frac{\bar{d}-s}{\sigma_{d}}\right)^{2}}\right)^{-1}
$$

Optimal demand (dotted line) when the signal constitutes a one standard deviation bad news surprise is shown in the left graph of Figure 9 and it is shown for a two standard deviation bad news surprise in the right graph. For comparison, I also plot the demand of two standard expected utility maximizers with dogmatic beliefs $\phi_{a}$ (dashed line) and $\phi_{b}$ (chain-dotted line), and demand of an ambiguity averse investor (solid line).

Optimal demand is a strictly decreasing function of the stock price and thus there is no portfolio inertia. If news is worse than expected, then investors think that it is more likely that the signal is unreliable and thus their demand resembles the demand of a standard expected utility maximizer with dogmatic belief $\phi_{a}$. Moreover, investors are worried about risk and thus hedge against uncertain information quality 
by putting less weight on the signal. However, for moderate long positions in the asset investors deviate from the demand of a standard expected utility maximizer with dogmatic belief $\phi_{a}$ because investor are more worried about a low posterior mean than risk when news is bad and thus they hedge by putting more weight on the signal.

\section{*** INSERT FIGURE 9 ABOUT HERE ***}

The equilibrium signal-to-price map when there is a standard expected utility maximizing representative investor with random signal precision is shown in Figure 10 (dashed line). This figure also shows the equilibrium signal-to-price map when there are two standard expected utility maximizing representative investors with dogmatic beliefs $\phi_{a}$ (dashed line) and $\phi_{b}$ (chain-dotted line), and it shows the equilibriumsignal-to-price map when the representative investor is averse to ambiguity (solid line). The equilibrium signal-to-price map is smooth when investors are standard expected utility maximizers and thus there is no excess volatility.

\section{*** INSERT FIGURE 10 ABOUT HERE ***}

\section{Conclusion}

In this paper I study the effects of risk and ambiguity on optimal portfolios and equilibrium asset prices when investors receive a signal with unknown precision. I show that desire of investors to hedge ambiguity leads to portfolio inertia for risky portfolios. Moreover, I show that small shocks to cash flow news, asset betas or market risk premia can lead to drastic changes in the worst case scenario belief about the precision of the signal and thus an econometrician observing the resulting drastic price change may conclude excess volatility. 


\section{A Proofs}

Let $\mu_{\phi}=\bar{d}+\phi(s-\bar{d})$ denote the posterior asset mean and $v_{\phi}=\sigma_{d}^{2}(1-\phi)$ the posterior asset variance. The next lemma is used for the proofs of Proposition 1 and 2.

Lemma 1. The posterior mean of future wealth $\tilde{w}$ given in equation (1) is

$$
\begin{aligned}
\mathrm{E}_{\phi}[\tilde{w} \mid \tilde{s}=s] & =w_{0}+\left(\mu_{\phi}(s)-p\right) \theta+\bar{L}+z\left(\mu_{\phi}(s)-\bar{d}\right) \\
& =w_{0}+\bar{L}+(p-\bar{d}) z+\left(\mu_{\phi}(s)-p\right)(\theta+z) .
\end{aligned}
$$

The posterior variance of future wealth $\tilde{w}$ given in equation (1) is

$$
\begin{aligned}
\operatorname{Var}_{\phi}[\tilde{w} \mid \tilde{s}=s] & =\operatorname{Var}_{\phi}\left[(\theta+z) \tilde{d}+\tilde{\varepsilon}_{L} \mid \tilde{s}=s\right] \\
& =v_{\phi}(\theta+z)^{2}+\sigma_{L}^{2}\left(1-\rho^{2}\right) .
\end{aligned}
$$

The certainty equivalent of a standard expected utility maximizer with belief $\phi$ is

$$
\begin{aligned}
C E^{S}(\theta, \phi) & =\mathrm{E}_{\phi}[\tilde{w} \mid \tilde{s}=s]-\frac{1}{2} \gamma \operatorname{Var}_{\phi}[\tilde{w} \mid \tilde{s}=s] \\
& =w_{0}+\bar{L}+(p-\bar{d}) z-\frac{1}{2} \gamma \sigma_{L}^{2}\left(1-\rho^{2}\right) \\
& +\left(\mu_{\phi}(s)-p\right)(\theta+z)-\frac{1}{2} \gamma v_{\phi}(\theta+z)^{2}
\end{aligned}
$$

Proof. To determine the posterior mean and variance of future wealth rewrite labor income as $\tilde{L}=\bar{L}+z(\tilde{d}-\bar{d})+\tilde{\varepsilon}_{L}$ with $z=\rho \sigma_{L} / \sigma_{d}$ and $\tilde{\varepsilon}_{L} \sim \mathrm{N}\left(0, \sigma_{L}^{2}\left(1-\rho^{2}\right)\right)$ and use the fact that $\tilde{\varepsilon}_{L}$ is independent of $\tilde{s}$.

It is well known that the certainty equivalent of a CARA utility investor with normally distributed wealth is

$$
\mathrm{CE}^{S}(\theta, \phi)=\mathrm{E}_{\phi}[\tilde{w} \mid \tilde{s}=s]-\frac{1}{2} \gamma \operatorname{Var}_{\phi}[\tilde{w} \mid \tilde{s}=s]
$$

Plugging equation (A.1) and (A.2) into equation (A.4) leads to the certainty equivalent in equation (A.3).

Proof of Proposition 1. The utility of an ambiguity averse investor given in equation (8) is

$$
\begin{aligned}
\min _{\phi \in\left[\phi_{a}, \phi_{b}\right]} \mathrm{E}_{\phi}\left[u\left(w_{0}+(\tilde{d}-p) \theta+\tilde{L}\right) \mid \tilde{s}=s\right] & =\min _{\phi \in\left[\phi_{a}, \phi_{b}\right]} u\left(\mathrm{CE}^{S}(\theta, \phi)\right) \\
& =u\left(\min _{\phi \in\left[\phi_{a}, \phi_{b}\right]} \operatorname{CE}^{S}(\theta, \phi)\right)
\end{aligned}
$$

where $\mathrm{CE}^{S}(\theta, \phi)$ is given in equation (A.3). Hence, the certainty equivalent of an 
ambiguity averse investor is

$$
\begin{aligned}
\mathrm{CE}(\theta) & =\min _{\phi \in\left[\phi_{a}, \phi_{b}\right]} \operatorname{CE}^{S}(\theta, \phi) \\
& =\min _{\phi \in\left[\phi_{a}, \phi_{b}\right]}\left(\mathrm{E}_{\phi}[\tilde{w} \mid \tilde{s}=s]-\frac{1}{2} \gamma \operatorname{Var}_{\phi}[\tilde{w} \mid \tilde{s}=s]\right) .
\end{aligned}
$$

To prove Proposition 1 we need to minimize $\mathrm{CE}^{S}(\theta, \phi)$ given in equation (A.3) w.r.t. $\phi$. Let $x=\theta+z \cdot \mathrm{CE}^{S}(x, \phi)$ does not depend on $\phi$ if $x=0$ and $x=\hat{\theta}$. Hence,

$$
\begin{aligned}
\mathrm{CE}(0) & =\min _{\phi \in\left[\phi_{a}, \phi_{b}\right]} \mathrm{CE}^{S}(0, \phi)=\mathrm{CE}^{S}\left(0, \phi_{a}\right)=\mathrm{CE}^{S}\left(0, \phi_{b}\right) \\
& =w_{0}+\bar{L}+(p-\bar{d}) z-\frac{1}{2} \gamma \sigma_{L}^{2}\left(1-\rho^{2}\right)
\end{aligned}
$$

and

$$
\begin{aligned}
\mathrm{CE}(\hat{\theta}) & =\min _{\phi \in\left[\phi_{a}, \phi_{b}\right]} \operatorname{CE}^{S}(\hat{\theta}, \phi)=\operatorname{CE}^{S}\left(\hat{\theta}, \phi_{a}\right)=\operatorname{CE}^{S}\left(\hat{\theta}, \phi_{b}\right) \\
& =w_{0}+\bar{L}+(p-\bar{d}) z-\frac{1}{2} \gamma \sigma_{L}^{2}\left(1-\rho^{2}\right)+2 \frac{\bar{d}-s}{\gamma \sigma_{d}^{2}}(s-p) .
\end{aligned}
$$

Taking the first derivative of $\mathrm{CE}^{S}(x, \phi)$ w.r.t. $\phi$ leads to

$$
\frac{\partial \mathrm{CE}^{S}(x, \phi)}{\partial \phi}=x(s-\bar{d})+\frac{1}{2} \gamma \sigma_{d}^{2} x^{2}=\frac{1}{2} \gamma \sigma_{d}^{2}(x-\hat{\theta}) x
$$

Let's consider three different cases: (i) $s=\bar{d} \Leftrightarrow \hat{\theta}=0$, (ii) $s<\bar{d} \Leftrightarrow \hat{\theta}>0$, and (iii) $s>\bar{d} \Leftrightarrow \hat{\theta}<0$.

(i) Let $x \in \mathcal{R}$ then $\frac{\partial \mathrm{CE}^{S}(x, \phi)}{\partial \phi}>0$ for all $\phi \in\left[\phi_{a}, \phi_{b}\right]$ and thus the minimum of $\mathrm{CE}^{S}(x, \phi)$ is attained at $\phi=\phi_{a}$. Hence,

$$
\mathrm{CE}(x)=\min _{\phi \in\left[\phi_{a}, \phi_{b}\right]} \mathrm{CE}^{S}(x, \phi)=\mathrm{CE}^{S}\left(x, \phi_{a}\right) \quad \forall x \in \mathcal{R} .
$$

$\mathrm{CE}^{S}\left(x, \phi_{a}\right)$ is continuously differentiable and concave for all $x \in \mathcal{R}$ and thus $\mathrm{CE}(x)$ is continuously differentiable and concave for all $x \in \mathcal{R}$.

(ii) Let $x<0$ or $x>\hat{\theta}$ then $\frac{\partial \mathrm{CE}^{S}(x, \phi)}{\partial \phi}>0$ for all $\phi \in\left[\phi_{a}, \phi_{b}\right]$ and thus the minimum of $\mathrm{CE}^{S}(x, \phi)$ is attained at $\phi=\phi_{a}$. Let $0<x<\hat{\theta}$ then $\frac{\partial \mathrm{CE}^{S}(x, \phi)}{\partial \phi}<0$ for all $\phi \in\left[\phi_{a}, \phi_{b}\right]$ and thus the minimum of $\mathrm{CE}^{S}(x, \phi)$ is attained at $\phi=\phi_{b}$. Let $x=0$ or $x=\hat{\theta}$ then $\mathrm{CE}(0)$ and $\mathrm{CE}(\hat{\theta})$ are given in equation (A.9) and (A.10), 
respectively. In this case $\frac{\partial \mathrm{CE}^{S}(x, \phi)}{\partial \phi}=0$ for all $\phi \in\left[\phi_{a}, \phi_{b}\right]$. Hence,

$$
\mathrm{CE}(x)= \begin{cases}\mathrm{CE}^{S}\left(x, \phi_{a}\right) & \text { if } x \leq 0 \\ \mathrm{CE}^{S}\left(x, \phi_{b}\right) & \text { if } 0<x<\hat{\theta} \\ \mathrm{CE}^{S}\left(x, \phi_{a}\right) & \text { if } x \geq \hat{\theta}\end{cases}
$$

$\mathrm{CE}(x)$ is continuous for all $x \in \mathcal{R}$ because $\mathrm{CE}^{S}(x, \phi)$ is continuous for all $x$ and $\phi$, and $\mathrm{CE}^{S}\left(\hat{\theta}, \phi_{a}\right)=\mathrm{CE}^{S}\left(\hat{\theta}, \phi_{b}\right)$ and $\mathrm{CE}^{S}\left(0, \phi_{a}\right)=\mathrm{CE}^{S}\left(0, \phi_{b}\right)$.

$\mathrm{CE}^{S}(x, \phi)$ is continuously differentiable for all $x \in \mathcal{R}$ and $\phi \in\left[\phi_{a}, \phi_{b}\right]$ and the second derivative of $\mathrm{CE}^{S}(x, \phi)$ w.r.t. $x$ is negative for all $x \in \mathcal{R}$ and $\phi \in\left[\phi_{a}, \phi_{b}\right]$. Hence, there is an open neighborhood for all $x \in \mathcal{R} \backslash\{0, \hat{\theta}\}$ such that $\mathrm{CE}(x)$ is continuously differentiable and the second derivative of $\mathrm{CE}^{S}(x)$ w.r.t. $x$ is negative.

To verify non-differentiability at $x=0$ and $x=\hat{\theta}$ and concavity of CE $(x)$ let's determine the left and right derivative of $\mathrm{CE}(x)$ at $x=\hat{\theta}$ and $x=0$ :

$$
\begin{aligned}
& \mathrm{CE}^{\prime+}(\hat{\theta}) \equiv \lim _{x \downarrow \hat{\theta}} \frac{\partial \mathrm{CE}(x)}{\partial x}=\bar{d}-p+2(s-\bar{d})-\phi_{a}(s-\bar{d}) \\
& \mathrm{CE}^{\prime-}(\hat{\theta}) \equiv \lim _{x \uparrow \hat{\theta}} \frac{\partial \mathrm{CE}(x)}{\partial x}=\bar{d}-p+2(s-\bar{d})-\phi_{b}(s-\bar{d}) \\
& \mathrm{CE}^{\prime+}(0)=\lim _{x \downarrow 0} \frac{\partial \mathrm{CE}(x)}{\partial x}=\bar{d}+\phi_{b}(s-\bar{d})-p \\
& \mathrm{CE}^{\prime-}(0)=\lim _{x \uparrow 0} \frac{\partial \mathrm{CE}(x)}{\partial x}=\bar{d}+\phi_{a}(s-\bar{d})-p .
\end{aligned}
$$

We have that $\mathrm{CE}^{\prime-}(\hat{\theta})>\mathrm{CE}^{\prime+}(\hat{\theta})$ and $\mathrm{CE}^{\prime-}(0)>\mathrm{CE}^{\prime+}(0)$. Hence, $\mathrm{CE}(x)$ is concave for all $x \in \mathcal{R}$ and differentiable for all $x \in \mathcal{R} \backslash\{0, \hat{\theta}\}$.

(iii) It is straightforward to show using the same arguments as in the previous case that

$$
\mathrm{CE}(x)= \begin{cases}\mathrm{CE}^{S}\left(x, \phi_{a}\right) & \text { if } \quad x \leq \hat{\theta} \\ \mathrm{CE}^{S}\left(x, \phi_{b}\right) & \text { if } \hat{\theta}<x<0 \\ \mathrm{CE}^{S}\left(x, \phi_{a}\right) & \text { if } x \geq 0\end{cases}
$$

and that $\mathrm{CE}(x)$ is continuous and concave. Moreover, $\mathrm{CE}(x)$ is differentiable for all $x \in \mathcal{R} \backslash\{0, \hat{\theta}\}$.

Proof of Proposition 2. To solve for the optimal demand $\theta_{\phi}(p)$ of a Savage investor with belief $\phi$ we need to maximize his certainty equivalent which is given in equation (A.3) with respect to $\theta$. 
Let $x=\theta+z$ and take the first derivative of $\mathrm{CE}^{S}(x)$ w.r.t. $x$ and set it equal to zero:

$$
\mu_{\phi}(s)-p-\gamma v_{\phi} x=0 .
$$

Solving for $x$ leads to $x=\left(\mu_{\phi}(s)-p\right) /\left(\gamma v_{\phi}\right)$. The certainty equivalent is concave and thus $\theta_{\phi}=\left(\mu_{\phi}(s)-p\right) /\left(\gamma v_{\phi}\right)-z$ is the unique maximum of $\mathrm{CE}^{S}(\theta)$.

Proof of Theorem 1. Consider three cases: (i) $s-\bar{d}=0 \Leftrightarrow \hat{\theta}=0$, (ii) $s-\bar{d}<0 \Leftrightarrow$ $\hat{\theta}>0$, and (iii) $s-\bar{d}>0 \Leftrightarrow \hat{\theta}<0$.

(i) If $s=\bar{d}$, then it follows from Proposition 1 that $\operatorname{CE}(\theta)=\operatorname{CE}^{S}\left(\theta, \phi_{a}\right)$ for all $\theta \in \mathcal{R}$ and thus it follows from Proposition 2 that $\theta(p)=\left(\mu_{a}(s)-p\right) /\left(\gamma v_{a}\right)-z$. Moreover, $\theta(p)$ is strictly decreasing in $p$.

(ii) If $s<\bar{d}$, then $C E(\theta)$ has two kinks. Let's maximize $C E(\theta)$ w.r.t. $x=\theta+z$. Consider the five subcases (a) $p<p_{1}=\mu_{a}(s)-\hat{\theta} \gamma v_{a}$, (b) $p_{1} \leq p \leq p_{2}=$ $\mu_{b}(s)-\hat{\theta} \gamma v_{b}$, (c) $p_{2}<p<p_{3}=\mu_{b}(s)$, (d) $p_{3} \leq p \leq p_{4}=\mu_{a}(s)$, and (e) $p>p_{4}$.

(ii)(a) We need to show that $x=x_{a} \equiv \frac{\mu_{a}(s)-p}{\gamma v_{a}}$ maximizes $\mathrm{CE}(x)$ for all $p<p 1$. We have that

$$
x_{a}=\frac{\mu_{a}(s)-p}{\gamma v_{a}}>\frac{\mu_{a}(s)-p_{1}}{\gamma v_{a}}=\hat{\theta} .
$$

Hence, there exists an $\epsilon>0$ s.t. $\mathrm{CE}(\xi)=\mathrm{CE}^{S}\left(\xi, \phi_{a}\right)$ for all $\xi \in\left(x_{a}-\right.$ $\left.\epsilon, x_{a}+\epsilon\right)$. From Proposition 2 follows that $x_{a}$ is a global maximum of $\mathrm{CE}^{S}\left(\xi, \phi_{a}\right)$ and thus $x_{a}$ maximizes $\mathrm{CE}(\xi)$ for all $\xi \in\left(x_{a}-\epsilon, x_{a}+\epsilon\right) \operatorname{CE}(\xi)$ is concave and thus $x_{a}$ is a global maximum of $\mathrm{CE}(\xi)$ for all $p<p 1$.

(ii)(b) We need to show that $x=\hat{\theta}$ maximizes $\mathrm{CE}(x)$ for all $p_{1} \leq p \leq p_{2}$. The certainty equivalent is concave (see Proposition 1) and hence it is sufficient to show that $\hat{\theta}$ is a local maximum. $\hat{\theta}$ is a local maximum if there is an $\epsilon>0$ such that

$$
\mathrm{CE}(\hat{\theta}) \geq \mathrm{CE}(x)=\min _{\phi \in\left[\phi_{a}, \phi_{b}\right]} \mathrm{CE}^{S}(x, \phi) \quad \forall x \in(\hat{\theta}-\epsilon, \hat{\theta}+\epsilon) .
$$

For $\epsilon$ sufficiently small we have that

$$
\mathrm{CE}(x)=\left\{\begin{array}{l}
\mathrm{CE}^{S}\left(x, \phi_{a}\right) \quad \text { if } \quad \hat{\theta} \leq x<\hat{\theta}+\epsilon \\
\mathrm{CE}^{S}\left(x, \phi_{b}\right) \text { if } \hat{\theta}-\epsilon<x \leq \hat{\theta}
\end{array}\right.
$$

Hence, we have to show that

$$
\begin{array}{ll}
\mathrm{CE}(\hat{\theta}) \geq \mathrm{CE}^{S}\left(x, \phi_{a}\right) & \forall x \in(\hat{\theta}, \hat{\theta}+\epsilon) \\
\mathrm{CE}(\hat{\theta}) \geq \mathrm{CE}^{S}\left(x, \phi_{b}\right) & \forall x \in(\hat{\theta}-\epsilon, \hat{\theta}) .
\end{array}
$$


Plugging in for both certainty equivalents in equation (A.23) we have for all $x \in[\hat{\theta}, \hat{\theta}+\epsilon)$ that $^{24}$

$$
\mathrm{CE}^{S}\left(x, \phi_{a}\right)-\mathrm{CE}(\hat{\theta})=(x-\hat{\theta})\left(\mu_{a}(s)-p-\hat{\theta} \gamma v_{a}-\frac{1}{2} \gamma v_{a}(x-\hat{\theta})\right) \leq 0
$$

Inequality (A.25) is satisfied for all $x \in[\hat{\theta}, \hat{\theta}+\epsilon)$ if

$$
\mu_{a}(s)-\hat{\theta} \gamma v_{a} \leq p
$$

Plugging in for both certainty equivalents in equation (A.24) we have for all $x \in(\hat{\theta}-\epsilon, \hat{\theta}]$ that $^{25}$

$$
\mathrm{CE}^{S}\left(x, \phi_{b}\right)-\mathrm{CE}(\hat{\theta})=(x-\hat{\theta})\left(\mu_{b}(s)-p-\hat{\theta} \gamma v_{b}-\frac{1}{2} \gamma v_{b}(x-\hat{\theta})\right) \leq 0 .
$$

Inequality (A.27) is satisfied for all $x \in(\hat{\theta}-\epsilon, \hat{\theta}]$ if

$$
p \leq \mu_{b}(s)-\hat{\theta} \gamma v_{b}
$$

Combining equations (A.26) and (A.28) leads to the desired result.

(ii)(c) We need to show that $x=x_{b} \equiv \frac{\mu_{b}(s)-p}{\gamma v_{b}}$ maximizes $\mathrm{CE}(x)$ for all $p_{2}<p<$ $p_{3}$. We have that

$$
\hat{\theta}=\frac{\mu_{b}(s)-p_{2}}{\gamma v_{b}}>x_{b}=\frac{\mu_{b}(s)-p}{\gamma v_{b}}>\frac{\mu_{b}(s)-p_{3}}{\gamma v_{b}}=0 .
$$

Hence, there exists an $\epsilon>0$ s.t. $\mathrm{CE}(x)=\mathrm{CE}^{S}\left(x, \phi_{b}\right)$ for all $x \in\left(x_{b}-\epsilon, x_{b}+\right.$ $\epsilon)$. From Proposition 2 follows that $x_{b}$ is a global maximum of $\mathrm{CE}^{S}\left(x, \phi_{b}\right)$ and thus $x_{b}$ maximizes $\mathrm{CE}(x)$ for all $x \in\left(x_{b}-\epsilon, x_{b}+\epsilon\right)$. $\mathrm{CE}(x)$ is concave and thus $x_{b}$ is a global maximum of $\mathrm{CE}(x)$ for all $p_{2}<p<p_{3}$.

(ii)(d) We need to show that $x=0$ maximizes $\mathrm{CE}(x)$ for all $p_{3} \leq p \leq p_{4}$. The certainty equivalent is concave (see Proposition 1) and hence it is sufficient to show that $x=0$ is a local maximum. $x=0$ is a local maximum if there is an $\epsilon>0$ such that

$$
\mathrm{CE}(0) \geq \mathrm{CE}(x)=\min _{\phi \in\left[\phi_{a}, \phi_{b}\right]} \mathrm{CE}^{S}(x, \phi) \quad \forall x \in(-\epsilon, \epsilon) .
$$

For $\epsilon$ sufficiently small we have that

$$
\mathrm{CE}(x)= \begin{cases}\mathrm{CE}^{S}\left(x, \phi_{b}\right) & \text { if } \quad 0 \leq x<\epsilon \\ \mathrm{CE}^{S}\left(x, \phi_{a}\right) & \text { if } \quad-\epsilon<x \leq 0 .\end{cases}
$$


Hence, we have to show that

$$
\begin{array}{ll}
\mathrm{CE}(0) \geq \mathrm{CE}^{S}\left(x, \phi_{b}\right) & \forall x \in[0, \epsilon) \\
\mathrm{CE}(0) \geq \mathrm{CE}^{S}\left(x, \phi_{a}\right) & \forall x \in(-\epsilon, 0] .
\end{array}
$$

Plugging in for both certainty equivalents in equation (A.32) we have for all $x \in[0, \epsilon)$ that $^{26}$

$$
\mathrm{CE}^{S}\left(x, \phi_{b}\right)-\mathrm{CE}(0)=x\left(\mu_{b}(s)-p-\frac{1}{2} \gamma v_{b} x\right) \leq 0 .
$$

Inequality (A.34) is satisfied for all $x \in[0, \epsilon)$ if

$$
\mu_{b}(s) \leq p
$$

Plugging in for both certainty equivalents in equation (A.33) we have for all $x \in(-\epsilon, 0]$ that $^{27}$

$$
\mathrm{CE}^{S}\left(x, \phi_{a}\right)-\mathrm{CE}(0)=x\left(\mu_{a}(s)-p-\frac{1}{2} \gamma v_{b} x\right) \leq 0
$$

Inequality (A.36) is satisfied for all $x \in(-\epsilon, 0]$ if

$$
p \leq \mu_{a}(s)
$$

Combining equations (A.35) and (A.37) leads to the desired result.

(ii)(e) We need to show that $x=x_{a} \equiv \frac{\mu_{a}(s)-p}{\gamma v_{a}}$ maximizes $\mathrm{CE}(x)$ for all $p>p 4$. We have that

$$
x_{a}=\frac{\mu_{a}(s)-p}{\gamma v_{a}}<\frac{\mu_{a}(s)-p_{4}}{\gamma v_{a}}=0 .
$$

Hence, there exists an $\epsilon>0$ s.t. $\mathrm{CE}(x)=\mathrm{CE}^{S}\left(x, \phi_{a}\right)$ for all $x \in\left(x_{a}-\epsilon, x_{a}+\right.$ $\epsilon)$. From Proposition 2 follows that $x_{a}$ is a global maximum of $\mathrm{CE}^{S}\left(x, \phi_{a}\right)$ and thus $x_{a}$ maximizes $\mathrm{CE}(x)$ for all $x \in\left(x_{a}-\epsilon, x_{a}+\epsilon\right)$. $\mathrm{CE}(x)$ is concave and thus $x_{a}$ is a global maximum of $\mathrm{CE}(x)$ for all $p>p 4$.

Demand $x(p)=\theta(p)+z$ and it is strictly decreasing in $p$ for the price ranges considered in (a), (c), and (e) and it is constant for the price ranges considered in (b) and (d) and hence $\theta(p)$ is a non increasing function of $p$.

(iii) If $s>\bar{d}$, then $C E(\theta)$ has two kinks. It is straightforward to show using the 
same arguments as in the previous case that

$$
\theta(p)=\left\{\begin{array}{lr}
\frac{\mu_{a}(s)-p}{\gamma v_{a}}-z & p \leq p_{1}=\mu_{a}(s) \\
-z & p_{1}<p \leq p_{2}=\mu_{b}(s) \\
\frac{\mu_{b}(s)-p}{\gamma v_{b}}-z & p_{2}<p \leq p_{3}=\mu_{b}(s)-\hat{\theta} \gamma v_{b} \\
\hat{\theta}-z & p_{3}<p \leq p_{4} \equiv \mu_{a}(s)-\hat{\theta} \gamma v_{a} \\
\frac{\mu_{a}(s)-p}{\gamma v_{a}}-z & p>p_{4} .
\end{array}\right.
$$

maximizes $\operatorname{CE}(\theta)$ for all $\theta \in \mathcal{R}$ and that $\theta(p)$ is non-increasing in the stock price $p$.

Proof of Proposition 3. It follows from Theorem 1 that the size of the price region at which investors exhibit portfolio inertia is $p_{2}-p_{1}$ and $p_{4}-p_{3}$. Specifically,

$$
\begin{aligned}
p_{2}-p_{1} & =\mu_{b}(s)-\mu_{a}(s)-\gamma\left(v_{b}-v_{a}\right) \max (\hat{\theta}, 0) \\
& =\left(\phi_{b}-\phi_{a}\right)(s-\bar{d})+\gamma \sigma_{d}^{2}\left(\phi_{b}-\phi_{a}\right) \max \left(2 \frac{\bar{d}-s}{\gamma \sigma_{d}^{2}}, 0\right) \\
& =\left(\phi_{b}-\phi_{a}\right)|s-\bar{d}|
\end{aligned}
$$

and

$$
\begin{aligned}
p_{4}-p_{3} & =\mu_{a}(s)-\mu_{b}(s)-\gamma\left(v_{a}-v_{b}\right) \min (\hat{\theta}, 0) \\
& =-\left(\phi_{b}-\phi_{a}\right)(s-\bar{d})-\gamma \sigma_{d}^{2}\left(\phi_{b}-\phi_{a}\right) \min \left(2 \frac{\bar{d}-s}{\gamma \sigma_{d}^{2}}, 0\right) \\
& =\left(\phi_{b}-\phi_{a}\right)|s-\bar{d}| .
\end{aligned}
$$

Proof of Proposition 4. From Proposition 2 follows that the demand of a Savage investor with belief $\phi$ is $\theta=\left(\mu_{\phi}(s)-p\right) /\left(\gamma v_{\phi}\right)-z$. The asset is in unit supply in equilibrium and thus $1=\left(\mu_{\phi}(s)-p\right) /\left(\gamma v_{\phi}\right)-z$. Solving for $p$ leads to equation (14).

Proof of Theorem 2. Take the optimal demand function given in equation (12) of Theorem 1 and set it equal to the market clearing stock demand of one.

Recall that $\hat{\theta}=2(\bar{d}-s) /\left(\gamma \sigma_{d}^{2}\right), \operatorname{Var}[\tilde{w}]=\sigma_{d}^{2}+2 \rho \sigma_{d} \sigma_{L}+\sigma_{L}^{2}>0, \beta=\left((1+z) \sigma_{d}^{2}\right) / \operatorname{Var}[\tilde{w}]$, and $\beta \lambda=\gamma \sigma_{d}^{2}(1+z)$. Consider the three cases (i) $z>-1 \Leftrightarrow \beta>0$, (i) $z<-1 \Leftrightarrow$ $\beta<0$, and (i) $z=-1 \Leftrightarrow \beta=0$. 
(i) Consider the three subcases (a) $s=\hat{s}$, (b) $s<\hat{s}$, and (c) $\hat{s}>s$ with $\hat{s}=$ $\bar{d}-\frac{1}{2} \gamma \sigma_{d}^{2}(1+z)<\bar{d}$.

(i)(a) Let

$$
\begin{aligned}
p_{a} & =\mu_{a}(\hat{s})-(1+z) \gamma v_{a} \\
p_{b} & =\mu_{b}(\hat{s})-(1+z) \gamma v_{b} .
\end{aligned}
$$

Then

$$
p_{b}-p_{a}=\left(\phi_{b}-\phi_{a}\right) \frac{1}{2} \gamma \sigma_{d}^{2}(1+z)>0
$$

and thus $\mathcal{P}_{\hat{s}}=\left[p_{a}, p_{b}\right]$. We need to verify that for all $p^{*} \in \mathcal{P}_{\hat{s}}$ we have that $\theta\left(p^{*}\right)=1$. If $s=\hat{s}$ then $\hat{\theta}=1+z>0$ and thus it follows from Theorem 1 that $\theta(p)=\hat{\theta}-z=1$ if and only if $p_{1} \leq p \leq p_{2}$ with

$$
\begin{aligned}
& p_{1}=\mu_{a}(\hat{s})-(1+z) \gamma v_{a} \\
& p_{2}=\mu_{b}(\hat{s})-(1+z) \gamma v_{b} .
\end{aligned}
$$

$p_{1}=p_{a}$ and $p_{2}=p_{b}$ and hence $\mathcal{P}_{\hat{s}}$ is the set of equilibrium prices.

(i) (b) We need to verify that $p^{*}(s)=\mu_{b}(s)-(1+z) \gamma v_{b}$ leads to $\theta\left(p^{*}(s)\right)=1$ for all $s<\hat{s}$. If $s<\hat{s}$ then $\hat{\theta}>1+z>0$ and thus it follows from Theorem 1 that

$$
\theta\left(p^{*}(s)\right)=\frac{\mu_{b}(s)-p^{*}(s)}{\gamma v_{b}}-z=1
$$

if and only if

$$
p_{2}=\mu_{b}(s)-\max (\hat{\theta}, 0) \gamma v_{b}<p^{*}(s)<\mu_{b}(s)-\min (\hat{\theta}, 0) \gamma v_{b}=p_{3} .
$$

Equation (A.48) is always satisfied because $\hat{\theta}>1+z>0$.

(i) (c) We need to verify that $p^{*}(s)=\mu_{a}(s)-(1+z) \gamma v_{a}$ leads to $\theta\left(p^{*}(s)\right)=1$ for all $s>\hat{s}$. If $s>\hat{s}$ then $\hat{\theta}<1+z$ and thus it follows from Theorem 1 that

$$
\theta\left(p^{*}(s)\right)=\frac{\mu_{a}(s)-p^{*}(s)}{\gamma v_{a}}-z=1
$$

if and only if

$$
p^{*}(s)=\mu_{a}(s)-(1+z) \gamma v_{a}<p_{1}=\mu_{a}(s)-\max (\hat{\theta}, 0) \gamma v_{a}
$$

or

$$
p^{*}(s)=\mu_{a}(s)-(1+z) \gamma v_{a}>p_{4}=\mu_{a}(s)-\min (\hat{\theta}, 0) \gamma v_{a} .
$$

Equation (A.50) is always satisfied because $(1+z)>\max (\hat{\theta}, 0)$. 
(ii) It is straightforward to show using the same arguments as in case (i) that

$$
p(s) \in \begin{cases}\left\{\mu_{a}(s)-(1+z) \gamma v_{a}\right\} & \text { if } \quad s<\hat{s} \\ \mathcal{P}_{\hat{s}} & \text { if } s=\hat{s} \\ \left\{\mu_{b}(s)-(1+z) \gamma v_{b}\right\} & \text { if } s>\hat{s}\end{cases}
$$

in which $\mathcal{P}_{\hat{s}}=\left[p_{b}, p_{a}\right]$ with

$$
\begin{aligned}
& p_{b}=\mu_{b}(\hat{s})-(1+z) \gamma v_{b} \\
& p_{a}=\mu_{a}(\hat{s})-(1+z) \gamma v_{a}
\end{aligned}
$$

and

$$
p_{a}-p_{b}=-\left(\phi_{b}-\phi_{a}\right) \frac{1}{2} \gamma \sigma_{d}^{2}(1+z)>0
$$

(iii) Consider the three subcases (a) $s=\bar{d}$, (b) $s<\bar{d}$, and (c) $s>\bar{d}$.

(iii)(a) Then it follows from Proposition 1 that $\operatorname{CE}(\theta)=\operatorname{CE}^{S}\left(\theta, \phi_{a}\right)$ for all $\theta \in \mathcal{R}$. From Proposition 4 follows that $p(s)=\bar{d}$ is the unique equilibrium price.

(iii)(b) Let $p_{a}=\mu_{a}(s)$ and $p_{b}=\mu_{b}(s)$ then

$$
p_{b}-p_{a}=\left(\phi_{b}-\phi_{a}\right)(s-\bar{d})<0
$$

and thus $\mathcal{P}_{0}(s)=\left[p_{b}, p_{a}\right]$. We need to verify that for all $p^{*} \in \mathcal{P}_{0}(s)$ we have that $\theta\left(p^{*}\right)=1$. If $s<\bar{d}$ then $\hat{\theta}>0$. From Theorem 1 follows that $\theta(p)=-z=1$ if and only if

$$
p_{3}=\mu_{b}(s) \leq p \leq p_{4}=\mu_{a}(s) .
$$

$p_{3}=p_{b}$ and $p_{4}=p_{a}$ and hence $\mathcal{P}_{0}(s)$ is the set of equilibrium prices.

(iii)(c) It is straightforward to show using the same arguments as in case (iii)(b) that $\theta(p)=-z=1$ if and only if

$$
p_{1}=\mu_{a}(s) \leq p \leq p_{2}=\mu_{b}(s)
$$

and hence $\mathcal{P}_{0}(s)=\left[p_{1}, p_{2}\right]$ is the set of equilibrium prices.

Proof of Proposition 5. The right and left limit of the price correspondence given in equation (18) are

$$
p^{+}(\hat{s})=\lim _{s \downarrow \hat{s}} p(s) \in \begin{cases}\left\{\bar{d}+\phi_{a}(\hat{s}-\bar{d})-\left(1-\phi_{a}\right) \beta \lambda\right\} & \text { if } \beta>0 \\ \mathcal{P}_{0}(\hat{s}) & \text { if } \beta=0 \\ \left\{\bar{d}+\phi_{b}(\hat{s}-\bar{d})-\left(1-\phi_{b}\right) \beta \lambda\right\} & \text { if } \beta<0\end{cases}
$$


and

$$
p^{-}(\hat{s}) \equiv \lim _{s \uparrow \hat{s}} p(s) \in \begin{cases}\left\{\bar{d}+\phi_{b}(\hat{s}-\bar{d})-\left(1-\phi_{b}\right) \beta \lambda\right\} & \text { if } \beta>0 \\ \mathcal{P}_{0}(\hat{s}) & \text { if } \beta=0 \\ \left\{\bar{d}+\phi_{a}(\hat{s}-\bar{d})-\left(1-\phi_{a}\right) \beta \lambda\right\} & \text { if } \beta<0\end{cases}
$$

respectively. It follows that

$$
\Delta p(\hat{s})=p^{+}(\hat{s})-p^{-}(\hat{s})=-\left(\phi_{b}-\phi_{a}\right) \frac{1}{2} \beta \lambda<0 \quad \text { if } \beta>0
$$

and

$$
\Delta p(\hat{s})=p^{+}(\hat{s})-p^{-}(\hat{s})=\left(\phi_{b}-\phi_{a}\right) \frac{1}{2} \beta \lambda<0 \quad \text { if } \beta<0 .
$$

The set of left price limits is identical to the set of right price limits if $\beta=0$ and thus

$$
\Delta p(\hat{s})=p^{+}(\hat{s})-p^{-}(\hat{s})=0
$$

It follows that

$$
\Delta p(\hat{s})=-\frac{1}{2}\left(\phi_{b}-\phi_{a}\right)(\max (\beta, 0)-\min (\beta, 0)) \lambda .
$$

Note that there is no discontinuity if investors are risk neutral $(\gamma=0)$ because in this case $\lambda=0$. There is no discontinuity if investors are standard expected utility maximizers because in this case $\phi_{a}=\phi_{b}$. Moreover, the price correspondence is upper hemicontinuous for all $\beta \lambda \in \mathcal{R}$ and $s \in \mathcal{R}$ but it is not lower hemicontinuous (and thus not continuous) for all $\beta \lambda \neq 0$ and $s \neq \bar{d}$.

Lemma 2 (Equilibrium Stock Price). Let $\gamma>0$ and $\operatorname{Var}[\tilde{w}]>0$. Define $\widehat{\beta \lambda}=$ $2(\bar{d}-s)$ and

$$
\begin{aligned}
& p_{a}(\beta \lambda)=\bar{d}+\phi_{a}(s-\bar{d})-\left(1-\phi_{a}\right) \beta \lambda \\
& p_{b}(\beta \lambda)=\bar{d}+\phi_{b}(s-\bar{d})-\left(1-\phi_{b}\right) \beta \lambda .
\end{aligned}
$$

Moreover, $p \in P_{\widehat{\beta \lambda}}$, if $\exists \phi \in\left[\phi_{a}, \phi_{b}\right]$ such that $p=\bar{d}+\phi(s-\bar{d})-(1-\phi) \widehat{\beta \lambda}$ and $p \in P_{0}$, if $\exists \phi \in\left[\phi_{a}, \phi_{b}\right]$ such that $p=\bar{d}+\phi(s-\bar{d})$.

Then there is a unique equilibrium stock price correspondence. Specifically, if $s<\bar{d}$, then

$$
p(\beta \lambda) \in \begin{cases}\left\{p_{a}(\beta \lambda)\right\} & \text { if } \beta \lambda>\widehat{\beta \lambda} \\ P_{\widehat{\beta \lambda}} & \text { if } \beta \lambda=\widehat{\beta \lambda} \\ \left\{p_{b}(\beta \lambda)\right\} & \text { if } 0<\beta \lambda<\widehat{\beta \lambda} \\ P_{0} & \text { if } \beta \lambda=0 \\ \left\{p_{a}(\beta \lambda)\right\} & \text { if } \beta \lambda<0 .\end{cases}
$$


If $s>\bar{d}$, then

$$
p(\beta \lambda) \in \begin{cases}\left\{p_{a}(\beta \lambda)\right\} & \text { if } \beta \lambda<\widehat{\beta \lambda} \\ P_{\widehat{\beta \lambda}} & \text { if } \beta \lambda=\widehat{\beta \lambda} \\ \left\{p_{b}(\beta \lambda)\right\} & \text { if } \widehat{\beta \lambda}<\beta \lambda<0 \\ P_{0} & \text { if } \beta \lambda=0 \\ \left\{p_{a}(\beta \lambda)\right\} & \text { if } \beta \lambda>0 .\end{cases}
$$

and if $s=\bar{d}$, then

$$
p(\beta \lambda)=p_{a}(\beta \lambda)
$$

I provide a proof of this Lemma in Section III of the internet appendix.

Proof of Proposition 6. The equilibrium price mapping $p(\beta \lambda)$ given in equation (A.67) and (A.68) has the two discontinuities $\beta \lambda=0$ and $\beta \lambda=\widehat{\beta \lambda}$.

It follows from equation (A.67), (A.68), and (A.69) that the right and left limit of the price mapping $p(\beta \lambda)$ when $\beta \lambda=\widehat{\beta \lambda}$ are

$$
p^{+}(\widehat{\beta \lambda})=\lim _{\beta \lambda \downarrow} p(\beta \lambda)=\left\{\begin{array}{lll}
p_{a}(\beta \lambda) & \text { if } & s \leq \bar{d} \\
p_{b}(\beta \lambda) & \text { if } & s>\bar{d}
\end{array}\right.
$$

and

$$
p^{-}(\widehat{\beta \lambda}) \equiv \lim _{\beta \lambda \uparrow \widehat{\beta \lambda}} p(s)=\left\{\begin{array}{lll}
p_{a}(\beta \lambda) & \text { if } & s \geq \bar{d} \\
p_{b}(\beta \lambda) & \text { if } & s<\bar{d}
\end{array}\right.
$$

respectively. Hence,

$$
\Delta p(\widehat{\beta \lambda})=p^{+}(\widehat{\beta \lambda})-p^{-}(\widehat{\beta \lambda})=\left\{\begin{array}{lll}
-\left(\phi_{b}-\phi_{a}\right)(\bar{d}-s) \leq 0 & \text { if } \quad s \leq \bar{d} \\
\left(\phi_{b}-\phi_{a}\right)(\bar{d}-s) \leq 0 & \text { if } s \geq \bar{d}
\end{array}\right.
$$

which verifies equation (20). It is straightforward to verify the size of the discontinuity given in equation (21) using similar arguments. Moreover, note that the price correspondence $p(\beta \lambda)$ is upper hemicontinuous for all $s \in \mathcal{R}$ but it is not lower hemicontinuous (and thus not continuous) for all $s \neq \bar{d}$. If $s=\bar{d}$ then $p(\beta \lambda)$ is a continuous function of $\beta \lambda$. 


\section{References}

Abel, Andrew, Janice Eberly, and Stavros Panageas, 2007, Optimal inattention to the stock market, American Economic Review 97, 244-249.

Ahn, David, Syngjoo Choi, Douglas Gale, and Shachar Kariv, 2010, Estimating ambiguity aversion in a portfolio choice experiment, Working Paper.

Allen, Franklin, and Douglas Gale, 1994, Limited market participation and volatility of asset prices, American Economic Review 84, 933-955.

Ameriks, John, and Stephen P. Zeldes, 2004, How do household portfolio shares vary with age, Working Paper.

Backus, David K., Bryan R. Routledge, and Stanley E. Zin, September 2004, Exotic preferences for macroeconomists, Working Paper.

Bewley, Truman F., 2002, Knightian decision theory. part i, Decisions in Economics and Finance: Springer 25, 79-110.

Billot, Antoine, Alain Chateauneuf, Itzhak Gilboa, and Jean-Marc Tallon, 2000, Sharing beliefs: Between agreeing and disagreeing, Econometrica 68, 685-694.

Bloom, Nicholas, 2009, The impact of uncertainty shocks, Econometrica 77, p. 623685.

Bossaerts, Peter, Paolo Ghirardato, Serena Guarnaschelli, and William Zame, 2010, Ambiguity in asset markets: Theory and experiment, Review of Financial Studies $23,1325-1359$.

Brennan, Michael J., and Yihong Xia, 2001, Stock price volatility and equity premium, Journal of Monetary Economics 47, 249-283. 
Caballero, Ricardo, and Arvind Krishnamurthy, 2008, Collective risk managment in a flight to quality episode, Journal of Finance LXIII, 2195-2230.

Cao, H. Henry, Tan Wang, and Harold H. Zhang, 2005, Model uncertainty, limited market participation, and asset prices, Review of Financial Studies 18, 1219-1251.

Caskey, Judson A., 2009, Information in equity markets with ambiguity-averse investors, Review of Financial Studies 22, 3595-3627.

Chapman, David A., and Valery Polkovnichenko, 2009, First-order risk aversion, heterogeneity, and asset market outcomes, Journal of Finance pp. 1863-1888.

Dow, J., and S. Werlang, 1992, Uncertainty aversion, risk aversion, and the optimal choice of portfolio, Econometrica 60, 197-204.

Dubinsky, Andrew, and Michael Johannes, 2005, Earnings announcements and equity options, Working Paper.

Easley, David, and Maureen O'Hara, 2009, Ambiguity and nonparticipation: The role of regulation, Review of Financial Studies 22, 1818-1843.

Easley, David, and Maureen O'Hara, 2010, Liquidity and valuation in an uncertain world, Journal of Financial Economics 97, 1-11.

Ellsberg, D., 1961, Risk, ambiguity, and the savage axioms, Quarterly Journal of Economics 75, 643-69.

Epstein, Larry G., and Martin Schneider, 2003, Recursive multiple-priors, Journal of Economic Theory 113, 1-31.

Epstein, Larry G., and Martin Schneider, 2007, Learning under ambiguity, Review of Economic Studies 74, 1275-1303. 
Epstein, Larry G., and Martin Schneider, 2008, Ambiguity, information quality, and asset pricing, Journal of Finance pp. 197-228.

Epstein, Larry G., and Martin Schneider, 2010, Ambiguity and asset markets, Annual Review of Financial Economics 2, 315-346.

Epstein, Larry G., and Tan Wang, 1994, Intertemporal asset pricing under Knightian uncertainty, Econometrica 62, 283-322.

Gilboa, Itzhak, and David Schmeidler, 1989, Maxmin expected utility with nonunique prior, Journal of Mathematical Economics 18, 141-153.

Gilboa, Itzhak, and David Schmeidler, 1993, Updating ambiguous beliefs, Journal of Economic Theory 59, p. 33-49.

Klibanoff, Peter, Massimo Marinacci, and Sujoy Mukerji, 2005, A smooth model of decison making under ambiguity, Econometrica 73, 1849-1892.

Knight, Frank H., 1921, Risk, Uncertainty, and Profit (Houghton Mifflin, Boston) 1 edn.

Paiella, Monica, 2007, The forgone gains of incomplete portfolios, Review of Financial Studies 20, p. 1623-1646.

Rigotti, Luca, Chris Shannon, and Tomasz Strzalecki, 2008, Subjective beliefs and ex ante trade, Econometrica 76, 1167-1190.

Routledge, Bryan R., and Stanley E. Zin, 2009, Model uncertainty and liquidity, Review of Economic Dynamics 12, 543-566.

Savage, Leonard J., 1954, The foundation of statistics (Wiley, New York) 1 edn.

Shiller, Robert J., 1992, Market Volatility (MIT Press) 1 edn. 
Veronesi, Pietro, 2000, How does information quality affect stock returns?, Journal of Finance pp. 807-837.

Vissing-Jorgensen, A., 2002, Limited asset market partipation and the elasticity of intertemporal substitution, Journal of Political Economy 110, 825-853.

Williams, Christopher D., 2009, Asymmetric responses to good and bad news: An empirical case for ambiguity, Working Paper.

Williamson, Stephen, 1994, Liquidity and market particpation, Journal of Economic Dynamics and Control 18, 629-670.

Yaron, Amir, and Harold Zhang, 2000, Fixed costs and asset market participation, Revista De Analisis Economico 15, 89-109.

Zweig, Jason, 2009, Why do investors sit tight in 401(k)s?, The Wall Street Journal p. B1. 


\section{Notes}

${ }^{1}$ Hence, this paper may shed some light on the fact that in the recent crisis investors didn't trade as much as one would expected in response to very bad news. "It is kind of striking," Mr. Utkus (from Vanguard) says. "We had the most drastic market decline since the Depression, we nearly had a total collapse of the global financial system, and all that caused most people not to do much at all." in Zweig (2009)

${ }^{2}$ Shiller (1992) writes in the introduction: "Prices change in substantial measure because the investing public en masse capriciously changes its mind".

${ }^{3}$ Epstein and Schneider (2008) consider an example in which the signal can take on two values and solve it numerically when investors are risk averse.

${ }^{4}$ The issue of observational equivalence is often raised in the literature (see Backus, Routledge, and Zin (September 2004)).

${ }^{5}$ Epstein and Schneider (2008) consider a simple experiment that describes how ambiguous information can lead to ambiguity about the posterior distribution even though the prior distribution is known. The analysis could be generalized to allow for an ambiguous prior without changing the main results of the paper. I show this in the internet appendix of this article which is available online in the Supplements and Datasets section at http://www.afajof.org/supplements.asp.

${ }^{6}$ Another updating rule that considers this rule as special cases is proposed in Epstein and Schneider (2007). See also Epstein and Schneider (2003) for updating 
Gilboa and Schmeidler (1989) preferences in dynamic models.

${ }^{7}$ The objective function is assumed to be continuous and the feasible set is compact and hence I can replace the infimum by the minimum.

${ }^{8}$ The posterior mean and residual variance of future wealth are given in equation (A.1) and (A.2) of the appendix.

${ }^{9}$ Easley and O'Hara (2009) consider investors with CARA utility who are ambiguous about the posterior mean and the variance of the asset. In their paper, investors always choose the highest possible variance and hence portfolio inertia only occurs at certainty.

${ }^{10}$ If the signal confirms the expected value of the dividend $(s=\bar{d})$, then there is no ambiguity about the conditional mean and hence the signal is always treated as unreliable.

${ }^{11}$ If ambiguity averse investors take on a short position in the asset, then they behave like Savage investor with belief $\phi_{a}$ because in this case the worst case scenario for the posterior asset mean and residual asset variances is an unreliable signal.

${ }^{12}$ If the signal conveys good news, then the risky portfolio that hedges against ambiguity is a short position in the asset.

${ }^{13} \mathrm{I}$ assume that there is uncertainty about aggregate wealth; i.e. $\operatorname{Var}[\tilde{w}]>0$.

${ }^{14}$ The equilibrium stock price also satisfies equation (18) if the representative investors is risk neutral ( $\gamma=0$ and thus $\lambda=0$ ). The proof is provided in Section II of 
the internet appendix.

${ }^{15}$ This behavior is empirically supported by Williams (2009) who shows that investors respond more to bad than to good earnings announcements following an increase in ambiguity.

${ }^{16} \mathrm{~A}$ signal value lower than $\hat{s}$ leads to more ambiguity about the posterior mean because it constitutes a larger news surprise. Hence, the portfolio $\hat{\theta}$ which hedges ambiguity is riskier for a signal value below $\hat{s}$ than above $\hat{s}$.

${ }^{17}$ The result is robust to other choices of $\phi$.

${ }^{18}$ The equilibrium stock price when there is no ambiguity about future labor income and investors are risk neutral and averse to ambiguity simplifies to (see Epstein and Schneider (2008))

$$
p(s)=\min _{\phi \in\left[\phi_{a}, \phi_{b}\right]} \mathrm{E}_{\phi}[\tilde{d} \mid \tilde{s}=s]=\bar{d}+\phi_{a} \max (s-\bar{d}, 0)+\phi_{b} \min (s-\bar{d}, 0) .
$$

${ }^{19}$ With slight abuse of notation I denote this mapping also with $p(\cdot)$ and I report it in Lemma 2 of the appendix.

${ }^{20}$ I provide formal proofs of results (i) and (ii) in Section IV of the internet appendix.

${ }^{21}$ The range of prices at which demand changes its slope does not depend on an investor's risk aversion and thus all demands can be added up as in the standard expected utility case. 
${ }^{22}$ There is also no price discontinuity if investors engage in betting or speculative trade because then they don't have a belief in common (see Billot, Chateauneuf, Gilboa, and Tallon (2000) or Rigotti, Shannon, and Strzalecki (2008)).

${ }^{23}$ I show in this section that there is no portfolio inertia and excess volatility by means of a simple example and refer the interested reader to Section $\mathrm{V}$ of the internet appendix for a rigorous proof.

${ }^{24}$ Inequality (A.23) is satisfied for all $x \in[\hat{\theta}, \hat{\theta}+\epsilon)$ if the right derivative of $\mathrm{CE}(x)$ evaluated at $x=\hat{\theta}$ is less or equal than zero.

${ }^{25}$ Inequality (A.24) is satisfied for all $x \in(\hat{\theta}-\epsilon, \hat{\theta}]$ if the left derivative of $\mathrm{CE}(x)$ evaluated at $x=\hat{\theta}$ is greater equal than zero.

${ }^{26}$ Inequality (A.32) is satisfied for all $x \in[0, \epsilon)$ if the right derivative of $\mathrm{CE}(x)$ evaluated at $x=0$ is less or equal than zero.

${ }^{27}$ Inequality (A.33) is satisfied for all $x \in(-\epsilon, 0]$ if the left derivative of $\mathrm{CE}(x)$ at $x=0$ is greater equal than zero. 

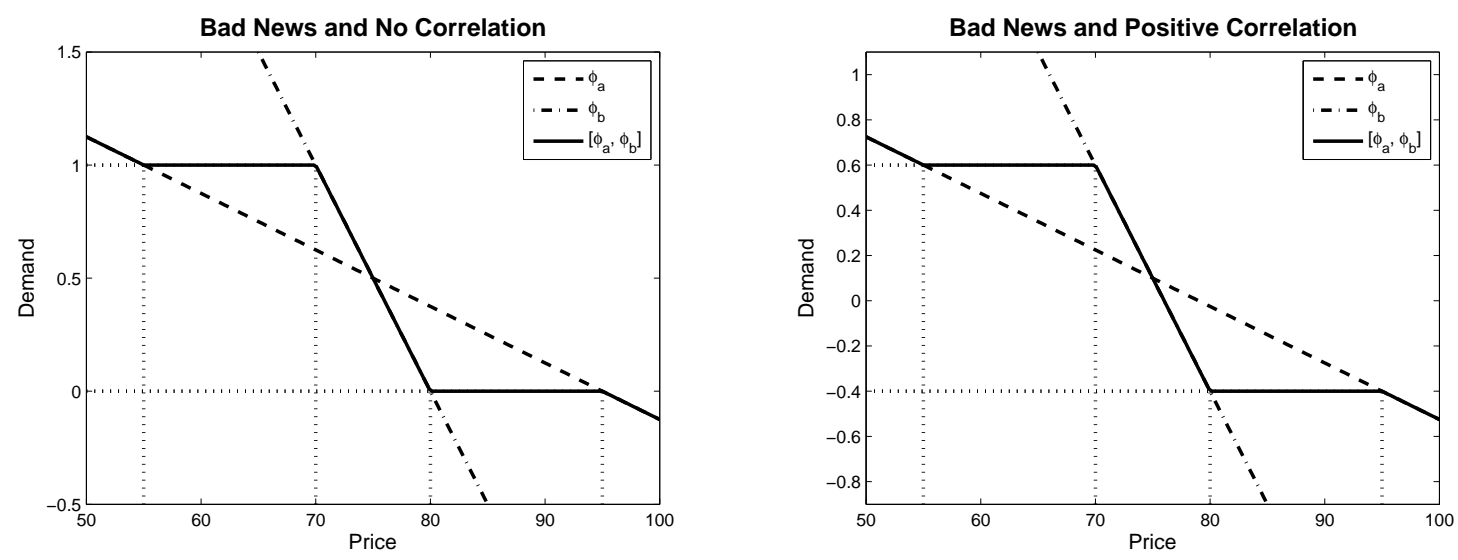

Figure 1: Optimal Demand

Both graphs show optimal demand when the signal conveys bad news $(s=75<$ $\bar{d}=100)$. The correlation between the stock and labor income is zero $(z=0)$ in the left graph and positive $(z=0.4)$ in the right graph. In both graphs demand is plotted as a function of the stock price for a Savage investor with belief $\phi_{b}$ (chain dotted line), for a Savage investor with belief $\phi_{a}$ (dashed line), and for an ambiguity averse investor with range of beliefs $\left[\phi_{a}, \phi_{b}\right]$ (solid line). There is a range of prices for which an ambiguity averse investor is neither long nor short the stock, does not change her long stock position, or does not change her short stock position. For all other prices her demand is equal to the demand of Savage investor with belief $\phi_{a}$ or $\phi_{b}$. The parameters are $\sigma_{d}=5, \gamma=2, \phi_{a}=1 / 5$, and $\phi_{b}=4 / 5$. 

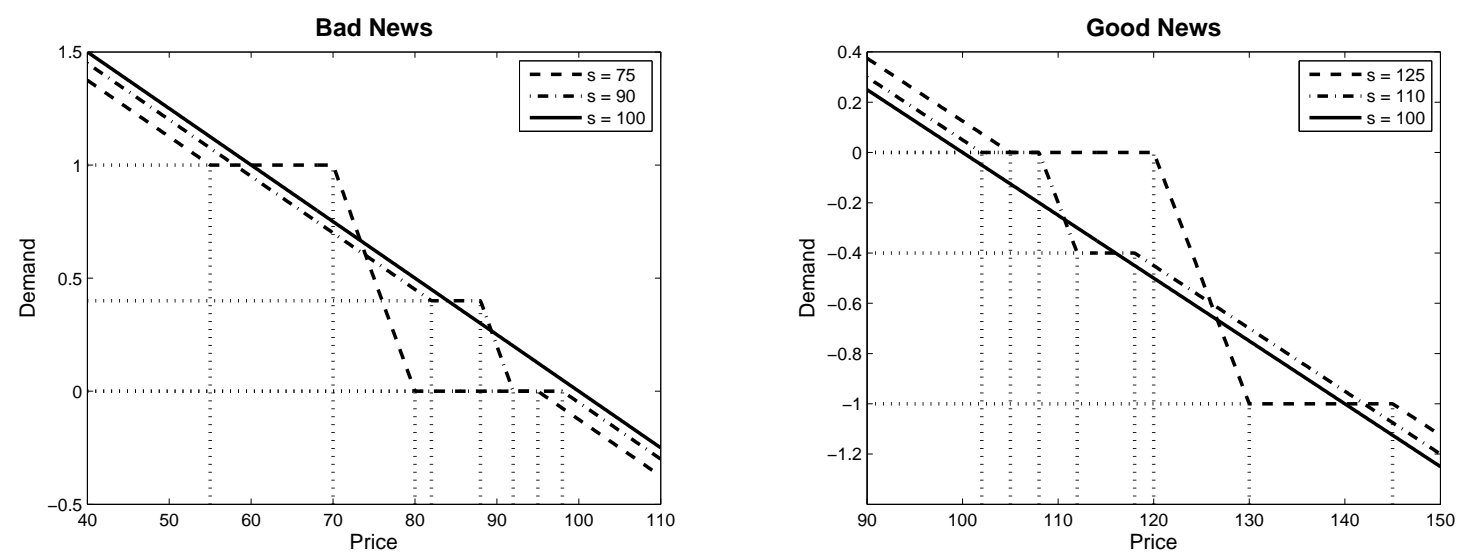

Figure 2: Portfolio Inertia

The left graph shows optimal demand of an ambiguity averse investor when news is bad and the right graph shows it when news is good. Demand is plotted as a function of the price for confirming news (solid line), a moderate news surprise (chain-dotted line), and for a big news surprise (dashed line). Both graphs show that when news is very surprising, then portfolio inertia is more severe and the portfolio at which investors exhibit inertia is more risky. The parameters are $\bar{d}=100, \sigma_{d}=5, \beta_{a}=1 / 5$, $\beta_{b}=4 / 5, z=0$, and $\gamma=2$. 

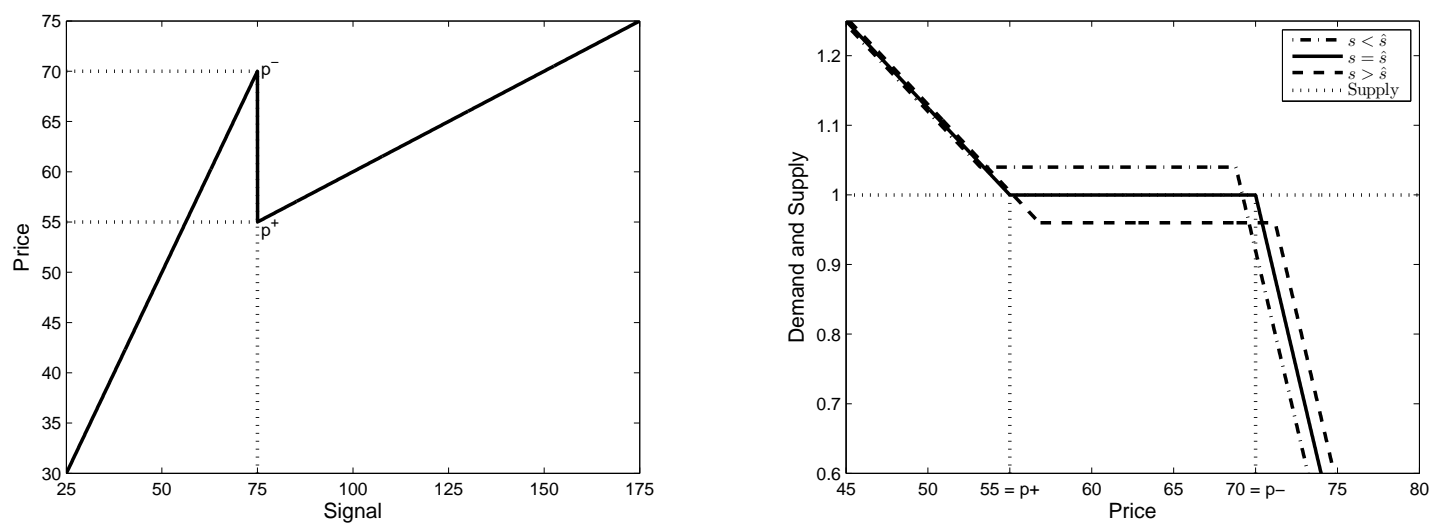

Figure 3: Equilibrium Stock Price

The left graph shows the equilibrium signal-to-price map of the stock. Investors treat the signal as reliable if $s<\hat{s}=75$ and as unreliable if $s>\hat{s}$. If the signal increases through $\hat{s}$ then the risk premium suddenly increases and thus the stock price drops. The right graph shows supply and demand as a function of the stock price for three different signal realizations. Supply is price inelastic and equal to demand for an interval of prices at $\hat{s}$. A slightly different signal realizations shifts the inaction region and thus leads to drastic changes in the market clearing stock price. The parameters are $\bar{d}=100, \sigma_{d}=5, \phi_{a}=1 / 5, \phi_{b}=4 / 5, \gamma=2$, and $\beta \lambda=\gamma \sigma_{d}^{2}=50$.
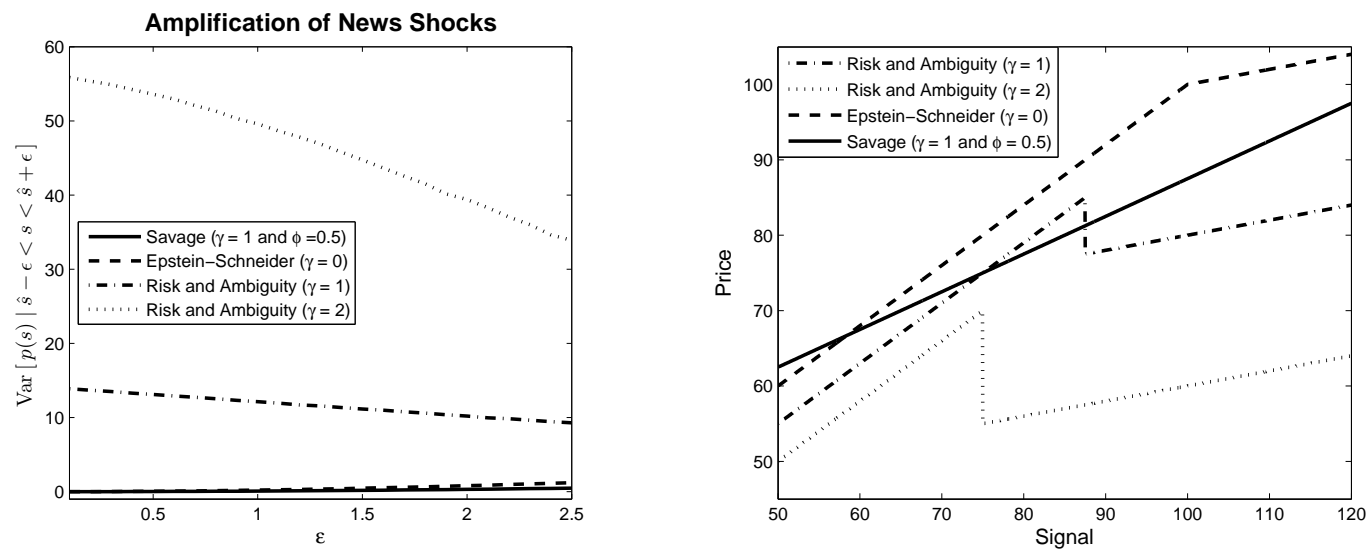

Figure 4: Shocks to Cash Flow News

The left graph shows the stock price variance conditional on the signal being in an $\epsilon$-intervall around the critical signal value $\hat{s}$. Small news shocks lead to a large variance when investors are averse to risk and ambiguity. The right graph shows the equilibrium signal-to-price map of the stock. The stock price has a discontinuity unless investors are neutral to ambiguity (solid line) or risk neutral (dashed line). The parameters are $\bar{d}=100, \sigma_{d}=5, \phi_{a}=1 / 5, \phi_{b}=4 / 5$, and $\lambda \beta=\gamma \sigma_{d}^{2}$. 

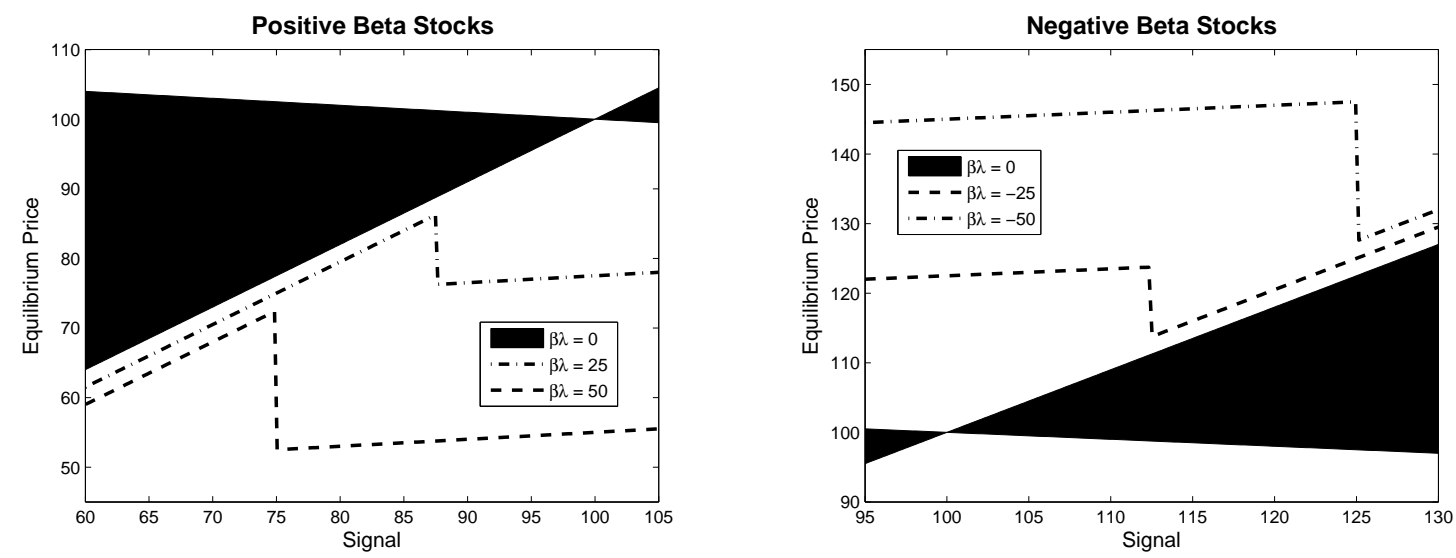

Figure 5: Positive and Negative Beta Stocks

Both graphs show the equilibrium signal-to-price map for different asset betas. There is a price discontinuity for bad news if the stock beta is positive and for good news if it is negative. The sudden price drops are more severe for risky stocks. The parameters are $\bar{d}=100, \sigma_{d}=5, \gamma=1, \phi_{a}=1 / 10$, and $\phi_{b}=9 / 10$.
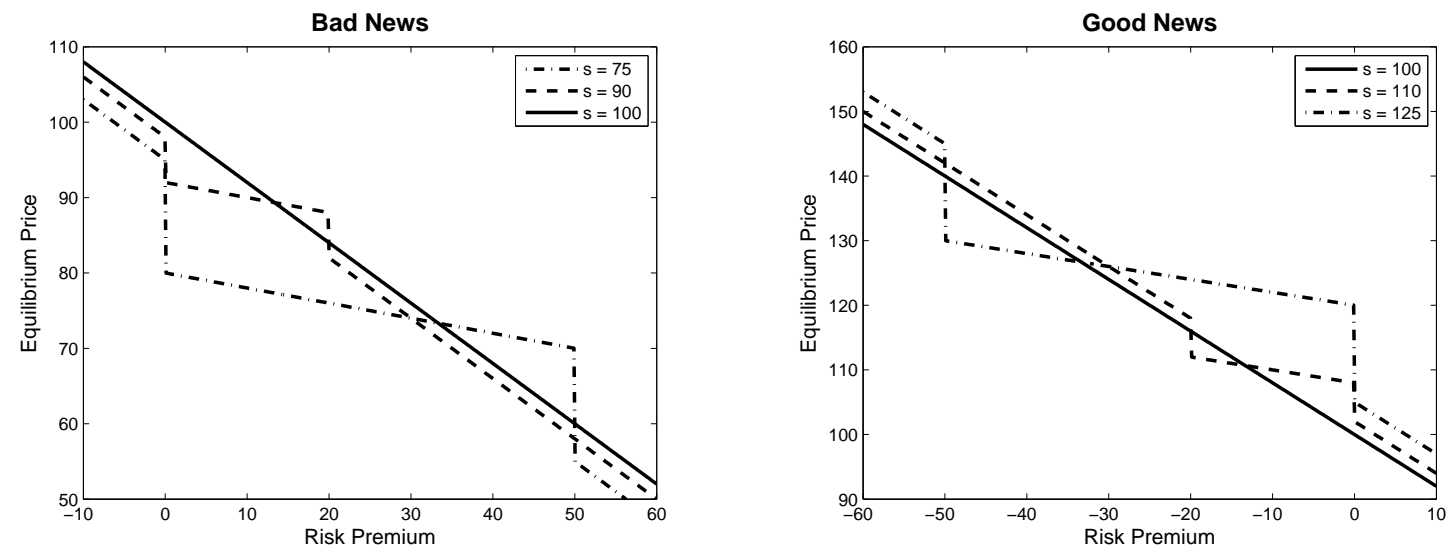

Figure 6: Shocks to Stock Risk Premia

Both graphs show the equilibrium risk-premium-to-price map. The signal conveys bad news in the left graph and good news in the right graph. Small changes to the stock risk premium may result in drastic changes in the stock price. The price drop is more severe for big news surprises. The parameters are $\bar{d}=100, \sigma_{d}=5, \gamma=1$, $\phi_{a}=1 / 10$, and $\phi_{b}=9 / 10$. 

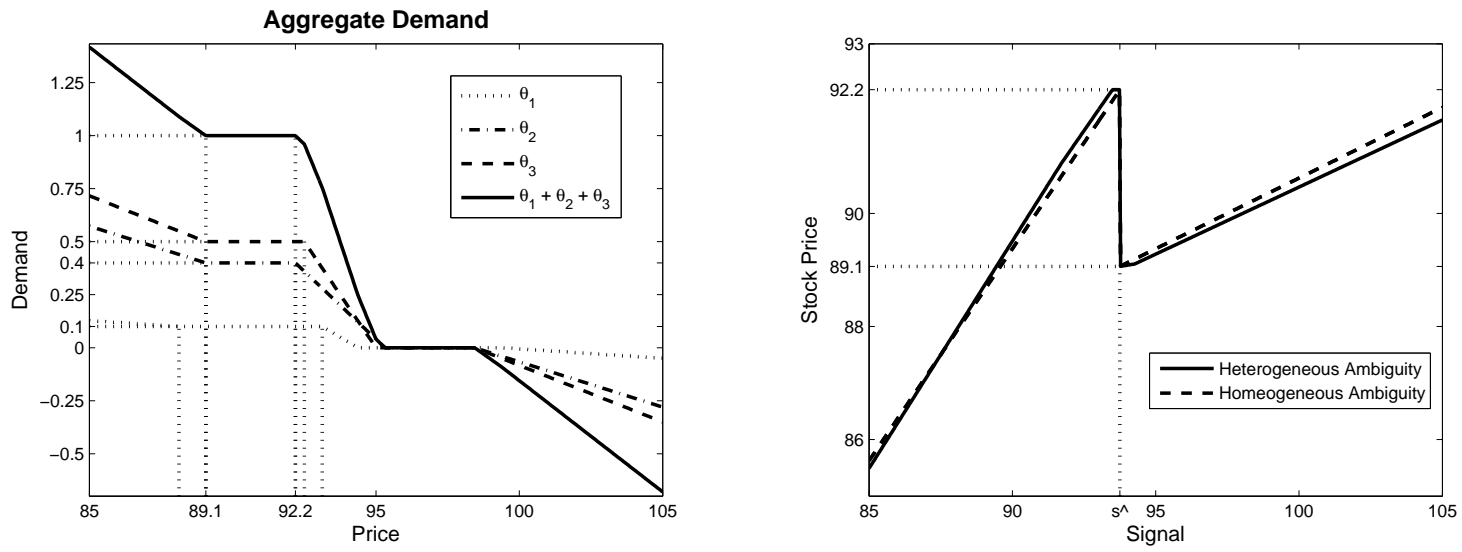

Figure 7: Aggregation

The left graph shows demand of three different investors and aggregate demand when $\hat{s}=\bar{d}-\frac{1}{2} \gamma \sigma_{d}^{2}=93.75$ with risk aversion equal to $\gamma=1 / 2$. Investors differ with respect to their aversion to risk and ambiguity: $\left(\theta_{1}\right) \gamma_{1}=5$ and $\left[\phi_{a 1}, \phi_{b 1}\right]=[1 / 10,9 / 10],\left(\theta_{2}\right)$ $\gamma_{2}=5 / 4$ and $\left[\phi_{a 2}, \phi_{b 2}\right]=[1 / 4,3 / 4]$, and $\left(\theta_{3}\right) \gamma_{3}=1$ and $\left[\phi_{a 3}, \phi_{b 3}\right]=[1 / 4,4 / 5]$. Individual demands add up to one for a range of prices at which the second investor does not change her demand and thus there is an interval of equilibrium stock prices. The right graph shows the equilibrium signal-to-price map for an economy that is populated by the three investors (solid line) and for a representative agent economy with $1 / \gamma=\sum_{h=1}^{3}\left(1 / \gamma_{h}\right)=1 / 2$ and $\left[\phi_{a}, \phi_{b}\right]=\bigcap_{h=1}^{3}\left[\phi_{a h}, \phi_{b h}\right]=[1 / 4,3 / 4]$ (dashed line). The parameters are $\bar{d}=100$ and $\sigma_{d}=5$. 

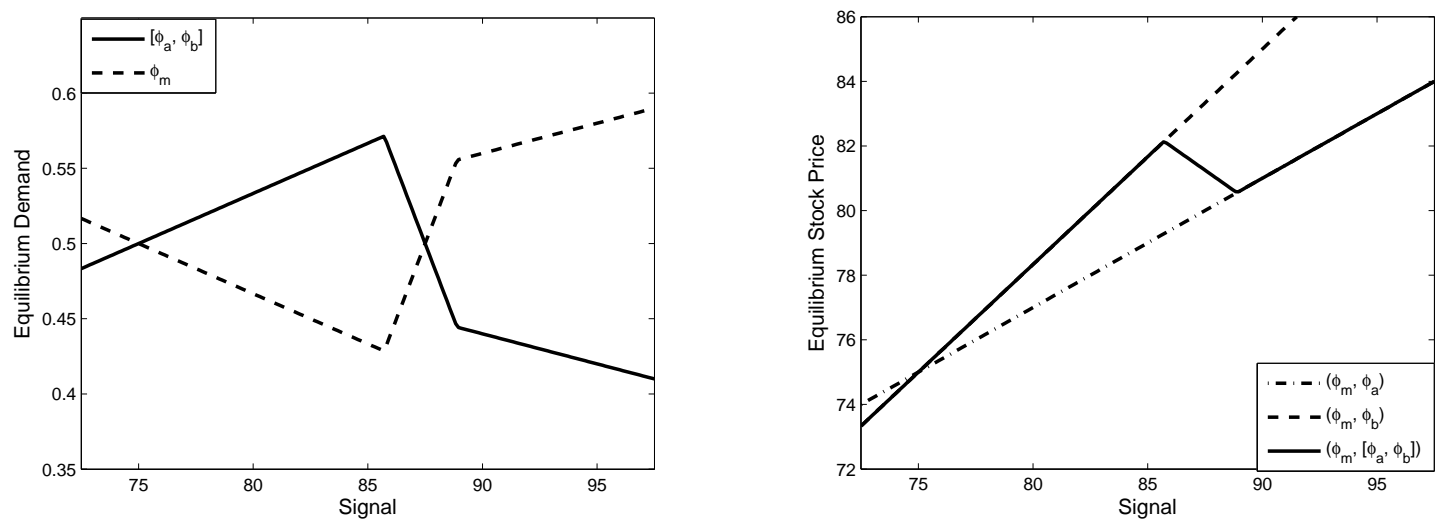

Figure 8: Ambiguity Averse and Savage Investors

The right graph shows the equilibrium price as a function of the signal when the economy consists of two Savage investors with beliefs $\phi_{m}=\left(\phi_{a}+\phi_{b}\right) / 2$ and $\phi_{b}$ (dashed line), two Savage investors with beliefs $\phi_{m}$ and $\phi_{a}$ (chain-dotted line), and one Savage investor with belief $\phi_{m}$ and one ambiguity averse investor with range of beliefs $\left[\phi_{a}, \phi_{b}\right]$ (solid line). The left graph shows optimal demand of the ambiguity averse investor (solid line) and the equilibrium demand of the Savage investor with belief $\phi_{m}$ (dashed line). There is a range of signal values for which (i) both investors are in the market and the ambiguity averse investor behaves like a Savage investor, (ii) both investors are in the market and the ambiguity averse investor does not behave like a Savage investor, (iii) the ambiguity averse investor does not participate (part (iii) is not apparent from the graphs but occurs for very good or bad news). 

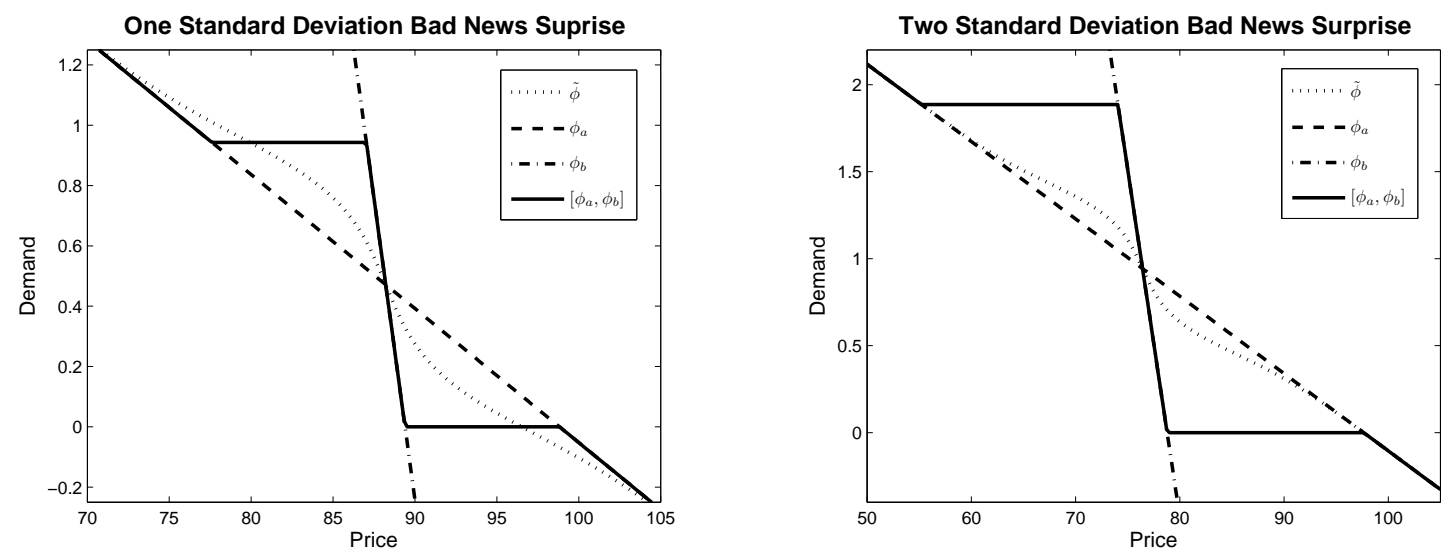

Figure 9: No Portfolio Inertia with Savage Investors

Both graphs show optimal demand of an ambiguity averse investor with a range of signal precisions $\left[\phi_{a}, \phi_{b}\right]$ (solid line), and investors with standard expected utility who have a unique prior over this range (dotted line), who have the dogmatic belief $\phi_{a}$ (dashed line), and who have the dogmatic belief $\phi_{b}$ (chain-dotted line). The left figure shows demand when the signal represents a one standard deviation news surprise $\left(\pi_{s}=75.47 \%\right)$ and the right figure shows it for a two standard deviation news surprise $\left(\pi_{s}=99.96 \%\right)$. Demands of standard expected utility maximizers are strictly decreasing in the stock price and thus there is no portfolio inertia. The parameters are $\bar{d}=100, \sigma_{d}=5, \gamma=1, \phi_{a}=1 / 10$, and $\phi_{b}=9 / 10$. 

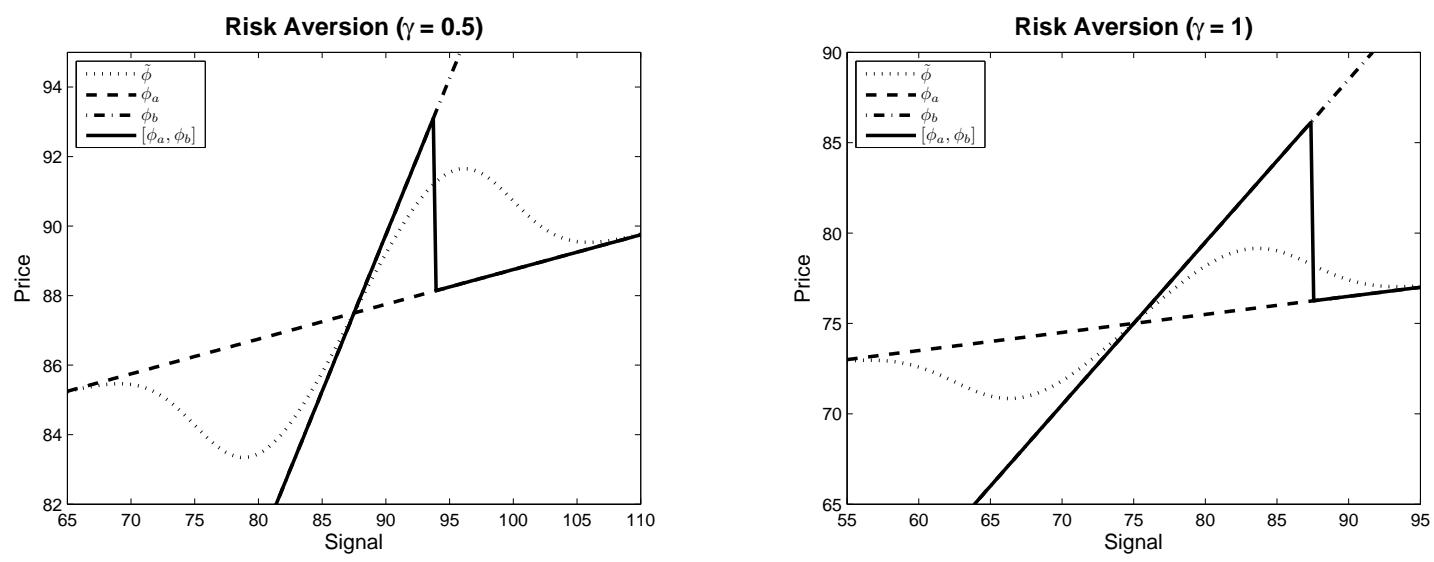

Figure 10: No Excess Volatility with Savage Investors

The left graph shows the equilibrium signal-to-price map when $\gamma=0.5$ and the right graph shows it when $\gamma=1$. In all graphs the price is plotted for an ambiguity averse representative investor who has a range of signal precisions $\left[\phi_{a}, \phi_{b}\right]$ (solid line), and for a representative investor with standard expected utility who has a unique prior over this range (dotted line), who has the dogmatic belief $\phi_{a}$ (dashed line), and who has the dogmatic belief $\phi_{b}$ (chain-dotted line). The stock price is a smooth function of the signal and thus there is no excess volatility when the representative investor maximizes standard expected utility. The parameters are $\bar{d}=100, \sigma_{d}=5, \phi_{a}=1 / 10$, and $\phi_{b}=9 / 10$. 